\title{
EVALUATION OF A VENTILATOR WITH FIXED \\ VOLUME CONTROL AND VARIABLE REGULATED PRESSURE 1, 2
}

\author{
Allen B Dobkin, M D, F A C A, D A (AM BD $)^{3}$
}

The many metabolic alterations which accompany wound trauma and anaesthesia have been studied by Bourne, Waters, Beecher, Moore, Holaday and others (1-10). These are the vital concern of the anaesthetıst because the changes due to the stress reaction are aggravated by hypoxıa and acidosis, which are usually the direct result of inadequate pulmonary ventilation.

The efficacy of automatic control bf pulmonary ventulation during anaesthesia in preventing hypoxia and acidosis has been the subject of wide discussion recently Many believe that automatic ventilators provide the anaesthetist and the surgeon with ideal conditıons, and prevent hypoxia and acidosis. However, manual control of ventilation provides more satisfactory conditions because it is accompanied by closer attention to the patient. The latter method of controlled ventilation should be continued until the automatic ventilators presently avallable can be adjusted quickly, so that inattention to the operative field is only required momentarily. Then changes in the lung or changes in the blood chemistry can be corrected as soon as detected (11-17)

The disadvantages of avallable automatic ventlators have encouraged renewed interest in those which are manually operated, which inform the anaesthetist continuously of the volume and pressure ampitude of pulmonary ventlation, and allow him to maintain some "feelng" of the airway. When better ventilators are developed, the function of the hand may be gradually relieved by a motor.

In 1899 Rudolph Matas employed a modified hand respirator, to produce artificial respiration through a laryngeal tube durng anaesthesia for thoracotomy (18). Since then many such devices have been employed for artificial respiration or resuscitation, but their application to clinical anaesthesia has been ignored It is surprising that, even in recent years, no simple hand bellows with volume calibration and a suitable pressure gauge hads been employed by anaesthetists.

Two hand ventrlators have been reported during the last few years. One was described recently by Poppelbaum in Germany (Fig. 1). Adequate controlled respiration is provided with this ventilator if the carbon dioxide content of the expired air is kept below 3 per cent This limitation requires regulation of several parameters of pulmonary ventilation as follows: minute volume, 6.0-9 0 litres, respiratory rate 12 to 20 per minute; the expiratory phase, at least two-thirds of the cycle; airway pressures, between +9 and $-5 \mathrm{~mm} \mathrm{Hg}$ when the chest is open, or +11 and $-5 \mathrm{~mm} \mathrm{Hg}$ in non-thoracic operations. These patients must be fully relaxed with ether anaesthesia and intermittent doses of a muscle

1Preliminary report was presented at the Canadian Aniaesthetssts' Society Annual Meetming in Saskatoon, Saskatchewan, June 26, 1957.

2This investigation was assisted by equipment and a grant-ın-ald from the Ohio Chemical and Surgical Company, Madison, Wisconsin.

${ }^{3}$ From the Department of Anaesthesia and the Cardiopulmonary Laboratory, University of Saskatchewan, College of Medicine and University Hospital, Saskatoon. 

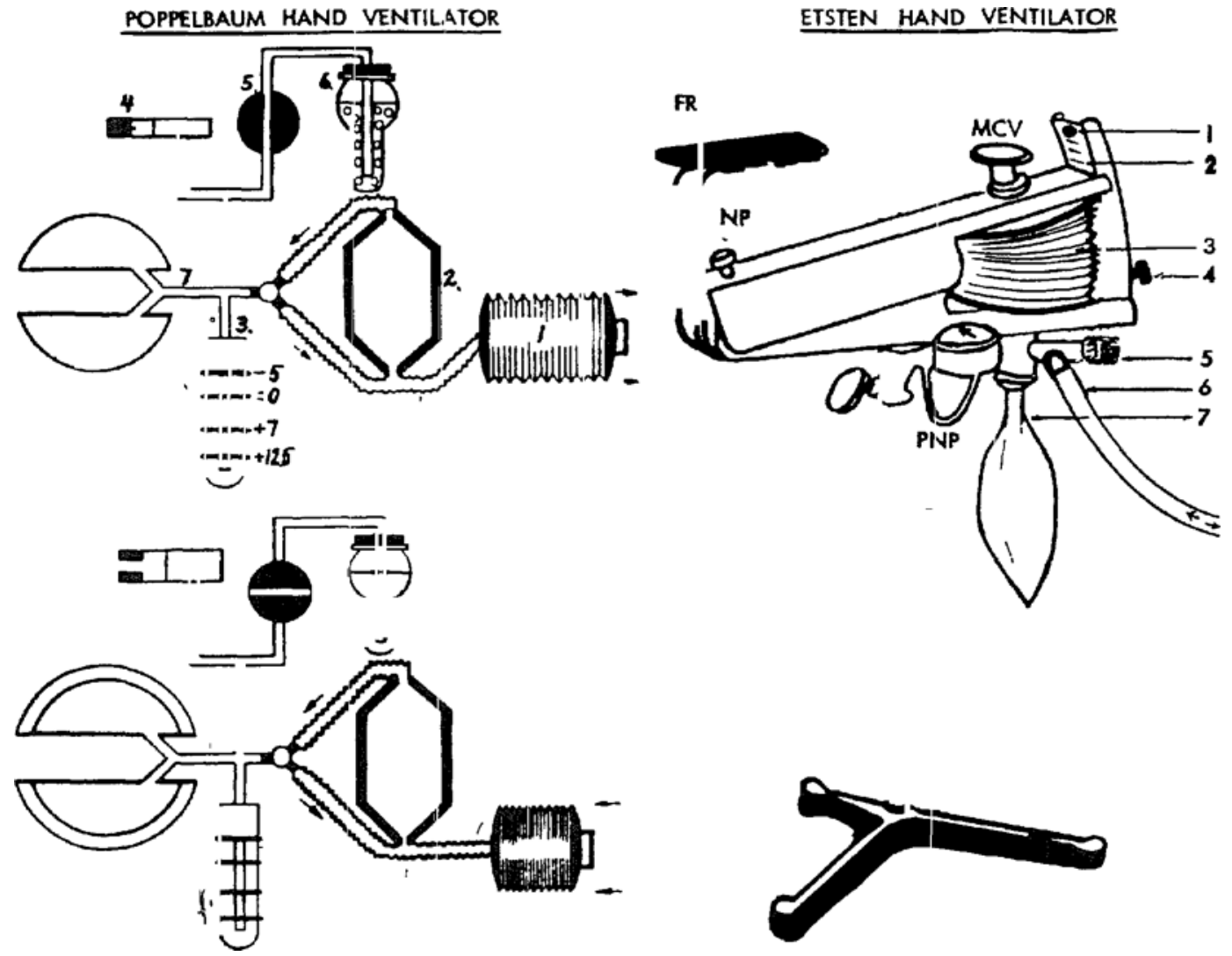

ETSTEN HAND VENTILATOR

Figure 1 Poppelbaum Hand Ventulator 1, volume calibrated hand bellows, 2, carbon dioxide absorber, 3, water pressure manometer; 4, relay switch, 5 , tap (opens duning expiration), 6, carbon dioxide indicator, 7 , endotracheal tube

Figure 2 Etsten Hand Ventulator 1, zero volume stop, 2, calibrated tidal volume scale, 3 , rubber accordion bellows, 4, volume set screw stop, 5, exhalation valve, 6, gas delivery tube, 7 , rebreathing bag attached to overllow vent on compensating valve, PNP, Marshalltown positive-ne yative pressure gauge, NP, negative pressure jlas screw adjustment, FR, forearm rest, MCV, manual -control lever.

relaxant. Durnng light anaesthesia, a greater volume and pressure were required to ventilate the lungs adequately (19).

MacKay uses a standard Coxeter-Mushin bellows hinged to the operating table to provide manual positive-negative pressure ventilation. Pressures of +15 to $-7 \mathrm{~mm}$. $\mathrm{Hg}$ were used during intrathoracic and upper abdominal operations The volume delivered was not measured, but occasional arterial blood $\mathrm{pH}$ determinations were made and found to exceed 7.5 (20).

Both authors were satisfied with their clinical results when these hand ventilators were used.

\section{Materiats AND Methods}

The following report deals with the clinical and laboratory evaluation of a more elegant hand-operated ventilator developed by Etsten. ${ }^{4}$ The outlet of this

${ }^{4}$ Available commercially, from the Ohio Chemical and Surgical Equpment Company. 
ventulator can be connected to a gas machine or directly to the patient's arway (see Fig. 2). The mechanical arrangements for use of this ventilator depended on the three anaesthetic systems which were studied.

\section{Non-Rebreathing System}

The manner in which the ventilator is modified when a non-rebreathing technique is used is shown in Figure 3 The rebreathing bag is removed from its
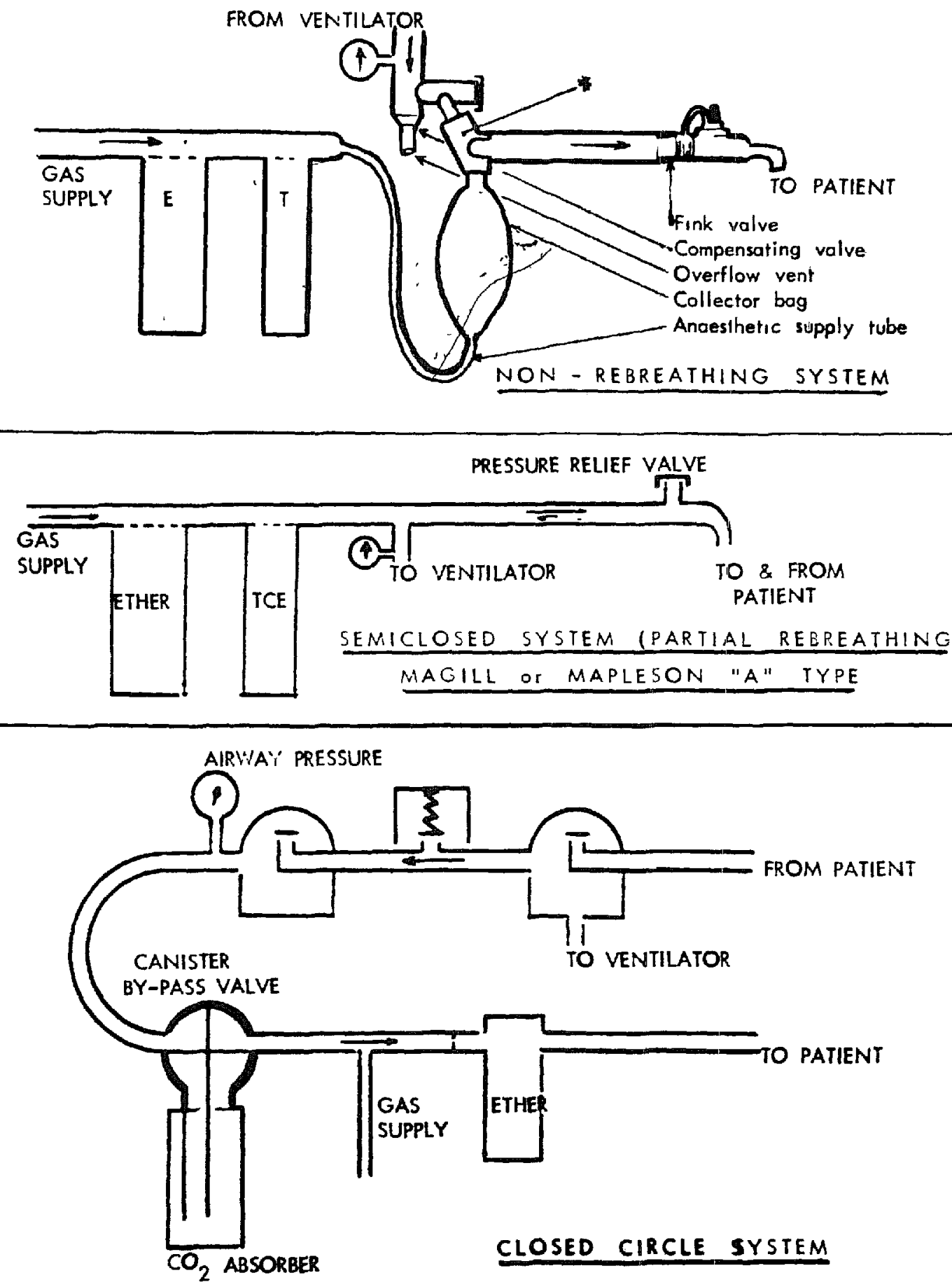

Figure 3 Anaesthetic systems 
nupple, which is then left open to atmosphere and serves as an overflow vent. A non-return valve labelled $\left({ }^{*}\right)$ is slipped over the outlet to which the delivery tubing is usually attached. The collector bag is attached to the vertical nipple of the non-return valve. The delivery tubing, which is equipped with a nonrebreathing valve of the Fink type at its free end, is attached to the horizontal mpple of the non-return valve. The fresh gas is delivered through the supply tubing to the collector bag, where the slight resistance of a check valve in the non-return assembly will cause it to be stored until the bellows is expanded to the point where it provides sufficient negative pressure to pull the contents of the collector bag through the check valve When the bellows is compressed, the

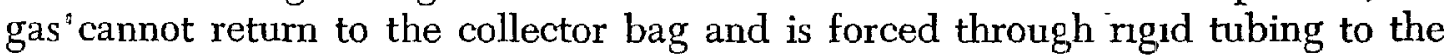
Fink valve, which is set so that the volumetric displacement of the bellows is added to the patient's lungs At the end of the inspratory stroke, the bellows rıșes, the expiratory diaphragm in the Fink valve opens, and the patient exhales to the atmosphere. At the same tume the bellows draws a fresh charge of gas from the collector bag The negative pressure (shown on the gauge) during expiration is transmitted only as far as the inhalation diaphragm of the Fink valve When the supply of gas from the source is in excess of that required to ventilate the patient, the collector bag fills slowly untıl gas is driven through the check valve of the non-return assembly and inflates the bellows sufficiently to open the compensatung valve of the ventlator. Thereater, the excess gas is vented to the atmosphere Venting the excess gas in this way acts as an automatic safety device. Simularly, should the supply of fresh gas be insufficient for the patient's needs to the point where the collector bag is emptued, the negative pressure in the system rises sufficiently to open the compensating valve and draws air through the overflow vent to make up what would otherwise be a ventilatory deficiency. This safety feature is likewise automatic

\section{Sema-closed System}

The ventilator is attached directly to the rebreathing bag nupple of the gas machine. The rebreathing bag is connected to the bellows of the ventilator through a compensating valve, in order to reduce the need for frequent adjustment of the gas inflow. Thus valve is open normally only when the bellows is at its maximum expansion It opens also when excessive negative pressure is generated within the bellows, as, for example, when the handle is forcibly raised or when there is a leak on the patient's side of the crrcuit. In this way the rebreathing bag serves as a reservoir which communicates with the breathing curcuit at the end of each cycle, and at all times when the bellows is at the top of its stroke. The rebreathing bag fills when the rate of gas flow exceeds the rate of utilization, and deflates if utilization exceeds inflow In either case, the anaesthetist becomes aware of the need for adjusting the delivery rate. At the usual flow rates employed with this system (8-14 L ), 175-200 ml. of gas are contributed to the set tidal volume by fresh gas when the duration of the inhalation phase of the breathing cycle is one-third of the respiration cycle. This volume is ignored when estimating the tidal volume setting in the Magill system (Mapleson A), because the corrugated tube from the gas machine to the patient expands during the inflation phase with a volume of gas simlar in amount. This "excess" 
volume begins to escape through the exhalation valve when inspiratory pressure is approaching its peak, and is completely lost during the exhalation phase. It was hoped also that this "excess" flow would reduce the amount of rebreathing that occurs in such a system when non-distensible tubes were used.

\section{Closed Circle System}

The ventulator is attached in the same way as for the semi-closed system If distensible corrugated tubing is used in thıs system, the volume of its expansion must be measured and added to the tidal volume settung.

If the occasion requires a "third hand," the manual lever may be driven automatically by a motor (double action pneumatic piston). The tidal volume and the duration of inspiration, expiration and rest phase can be adjusted within certain limits, each by a knurled nut on the face of the motor. When the maximum tidal volume adjustment of one litre is set, the expiration phase at atmospheric pressure is limited to one-third of the total cycle. As the tidal volume is reduced to the usual clinical range $(400-650 \mathrm{ml}$ ) a much longer expiration phase may be provided (greater than two-thirds). When manual control is once again feasible or desirable, the motor may be swung asıde on the supporting stand.

The clinical efficacy of the Etsten Ventilator has been studied in 332 patsents during general anaesthesia for major operations These patients fall into four main groups as shown in Table I In the first group, there were 25 patients who were placed in the lateral flexed position for intrathoracic operations. For most the non-rebreathing technique was employed. In the second group, there were 60 patients who were placed in the prone-jackknife position with the shoulder gurdle and the liac crest supported on a mattress as described by Bardeen (21) to allow free mobility of the chest and abdomen durmg operations on the spmal column (spinal cord tumor, spinal fusion and discoidectomy) In the third group, there were 132 patients who were placed in the supune horizontal position for upper abdominal and major superficial operations. The fourth group contamed 115 patients who were placed supine and in varying degrees of head low, or Trendelenburg position $\left(5-15^{\circ}\right)$, on a Hewer mattress.

For each patient the following were noted: body surface area in square metres, derived from the measured height and weight, body buld, coded as medium when the height, weight and age of the patient were withm the normal weight limits as listed by the Life Extension Institute (New York City); the underweight patient was coded as lean, and the overweight patient as obese, respectively; and the physical state was coded as 1 to 7 , according to the method recommended by the American Society of Anesthesiology. The majonty of patients were premedicated with meperidine and-either scopolamine or atropine. Others received ataractic drugs (Phenergan ${ }^{\circledR}$, Sparme $^{\circledR}$, Stemetil ${ }^{\otimes}$, or Trilafon ${ }^{\circledR}$ ) All premedicant drugs were administered intramuscularly, approximately one hour before induction of anaesthesia.

Anaesthesia was induced with a muscle relaxant, and a sleep dose of thiopental, followed by intubation with the largest cuffed endotracheal tube which would pass with ease. It was maintained at the lightest level consistent with the 


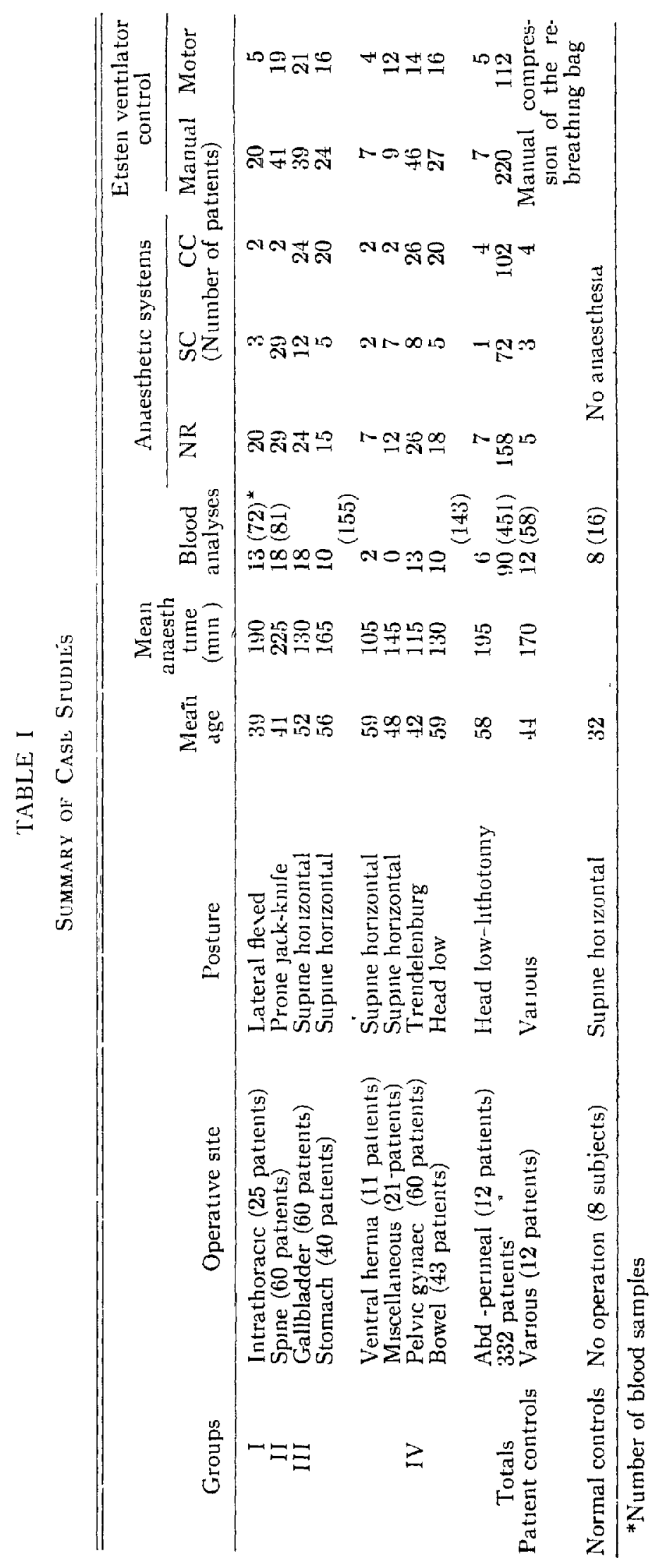


operative procedure, employing a balanced technique When cautery was used, the pumary agent was nitrous oxide-oxygen (2.1) with total flows set usually at 12 litres, supplemented by meperidine intravenously ard trichlorethylene in a semi-closed system of the Magill type (Mapleson A) or in a non-rebreathing system. Otherwise cyclopropane was used with a tiace of ether in a circle closed system or nitrous oxide was used with a trace of ether in a semi-closed system

The main object of this study was to test the prediction of a respiratory rate, volume and pressure amplitude which would maintain the arterial blood at a $\mathrm{pCO}_{2}$ near $40 \mathrm{~mm}$. Hg and a $\mathrm{pH}$ near $7.35^{5}$ Arterial blood samples were therefore obtained for analysis from the brachial artery in 90 patients immediately before induction of anaesthesia, at approximately half-hour intervals during anaesthesia (when possible), just prior to its termination, and within 30 minutes of discontinuing the anaesthetic

During the period of anaesthesia 5 per cent dextrose in water and whole blood transfusions were administered as was found necessary, according to weighed or estimated losses. No base was admmistered to any of these patients during the anaesthetic period or untll the last blood sample was clrawn so that the blood data observed represented the patrents' unmodified respiratory' and metabohc responses to ventilation and the anaesthetics The patients' body temperatures were not altered intentionally from normal levels in any of these operations in order to avoid gross errors in the constants for determining changés in the blood chemistry.

Each arterial blood sample was drawn anaerobically into specially prepared $10 \mathrm{ml}$. heparinized syringes After gentle mixing, alıquots of the samples were analysed in duplicate as follows. The $\mathrm{pH}$ was measured immediately (or withm two hours if the blood was iced) at room temperature, in a Cambridge glass electrode $\mathrm{pH}$ meter and corrected to body temperature $\left(37^{\circ} \mathrm{C}\right)(23,24)$. The haematocrit was determined by centrifuging the blood in a Wintrobe tube at $2,000 \mathrm{rpm}$. for 30 minutes The oxygen capacity of the blood was determined directly by equilibration in room aur for 15 minutes, according to Roughton et al. (25). The oxygen capacity was corrected for the effect of temperature of the Van Slyke apparatus on the dissolved oxygen. The haemoglobin content was calculated from: corrected oxygen capacty / 1.34 . The oxygen and carbon dioxide content of the whole blood were determined by vacuum extraction and manometric measurement $(26,27,28)$. The total carbon dioxide content of the plasma was calculated from the total $\mathrm{CO}_{2}$, haematocrit and $\mathrm{pH}$, according to Van Slyke and Sendroy (29) The oxygen tension was derived frorn Dill's nomogram (30). The percentage of oxygen saturation was calculated from content/capacity and was also determmed on whole haemolyzed blood, using a lucite cuvette adapted to the Beckman model D U Spectrophotometer, according to the Hickman and Frayser modification of the Nahas technique (31,32). These two methods for determining oxygen saturation were comparable ( $\mathrm{SD}= \pm 1$ per cent). (33). The carbon dioxpde tension in arterial blood and the bicarbonate concentration in the plasma were calculated from the formula derived from the HendersonHasselbalch equation $(34,35,36)$. From each analysis of blood gas which was

5 These are the approximate "normal" values found in normal subjects by our laboratory. 
carried out, the $\mathrm{pH}$ (the abscissa) and the bicarbonate concentration (the ordinate) were graphed, according to the method of Davenport (35)

Controlled respiration with the Etsten ventilator was manual in 220 cases and automatic $\mathrm{m} 112$ The characterstics of the ventilation cycle (volume and pressure), were determmed according to the nomal weight applied to Radford's nomogram and with further allowance for abnormal postures and pulmonary emphysema (37, 38) The volume setting was checked on occasion during anaesthesicl by incorporating the Bennett respiratory ventilation meter with rezero and anaesthesia bypass system (Model 104B) just proumal to the endotracheal tube (see Fig 4) The rate of respuration was adjusted to 18 per minute in all but a few cases, with the inspiratory phase approxmately 1 second in duration and an inspiratory peak pressure of $15 \mathrm{~mm}$ of $\mathrm{Hg}$ The evpuratory phase lasted appioumately 2 seconds with the mitial fracton $(04$ to I sec $)$ of this phase adjusted to $2 \mathrm{~mm}$ below atmospheric pressure and the remander of the cycle at atmosphonc pressure These claracteustics of the ventation cycle assumed that the inspuatory How rate (30 to 55 litres per minute) was sufficient to provide the range of inspuratory volume desured $(350$ to $750 \mathrm{ml})$, and could

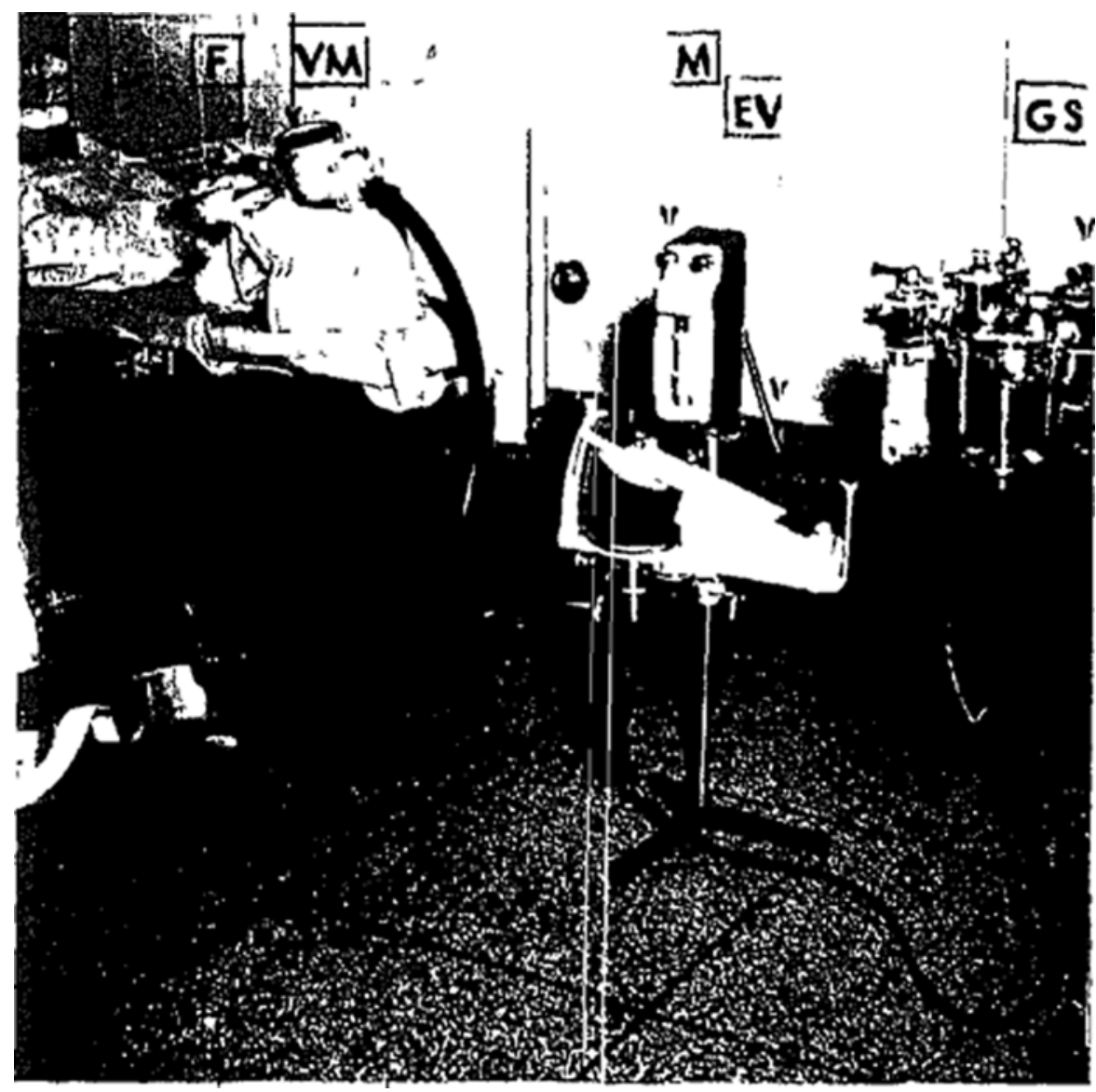

Frcune 4 Clmical arrangement of appantus F, Fink-type non rebreathing vilve, VM, Bemett ventilition meter (Sodel 104B), M, motor-driven by compressed aur or oxygen (non-explosive), EV, Etsten ventilator, GS, gis rupply (Nitrous oude-ouygen) directed through trichlorethylene in the nonrebreathing eircut of a Heidbrink ancest jetic machine 
be delivered in one second and removed in two seconds through the endotracheal tube by the ventilator. In order to prevent any loss of the volume in the external anaesthetic circuit, the gases were cycled through non-distensible tubıng in every case.

\section{RESULTS}

A condensed summary of the results of the blood analyses appears in Table II. Protocol data not essential to the discussion were deleted from this table to conserve space. The variation (standard deviation of the mean) observed in the blood data was analysed. This variation was compared with that of eight normal, healthy, unpremedicated subjects from whom two separate arterial blood samples were each analysed in duplicate to determine "normal values" observed in our laboratory. (Table III), and with arterial blood studies on twelve patients whose respiration was controlled by manual compression of the rebreathing bag. The Fusher-t test was applied to the blood data from each patient to determine whether the probability " $\mathrm{p}$ " of the differences observed under the conditions of the study could be due to chance alone (Table IV)

\section{Blood Data}

Intrathoracic operations. During the course of 13 of the 20 operations in the lateral flexed position employing the non-rebreathung system, 72 artenal blood samples were analysed. Regardless of the patient's age, general condition, or disease process in the lungs, there was full oxygen saturation of the blood, adequate removal of carbon dioxide and no significant rise in the fixed acids (see Figs. 5, 6 and 7). In no patient was there gross alteration in the blood pressure or pulse rate due to the anaesthetsc method or agents. In some there was a slight elevation of the blood pressure untıl the pleura was opened and then it returned to the preoperative level. This is the reverse of what is usually seen with manual compression of the rebreathing bag. When the patient was allowed to breath spontaneously after the pleura was closed, the slight alkalosis, which had developed during controlled respiration, disap reared promptly There was no marked tendency toward a respiratory acidoses but hypoxia could only be avoided by continuing the administration of 100 per cent oxygen by mask or nasal catheter during the early postanaesthetic period. Recovery from anaesthetic was prompt and in none of these patients was there an alarming depression of the vital signs. The other five thoracotomy patients were managed by the semi-closed system ( 3 cases) and the closed circle system ( 2 cases) without clinical difficulty. No blood data were obtained from these. In long intrathoracic operations, manual compression of the rebreathing bag was not as efficient as controlled respiration with the ventilator (see Fig: 8).

Spinal operations. During 18 of the 60 operations in the prone jackknife position, 81 arterial blood samples were analysed. The non-rebreathing system was employed in 29 patients. Blood data obtained from 9 of these revealed that there was no significant alteration in the $\mathrm{pH}$ or carbon dioxide tension (see Figs. 9 and 10), except in one patient (S-22). In the latter case, the change was due to a technical defect (see Discussion). The semi-closed system of the Magill type 


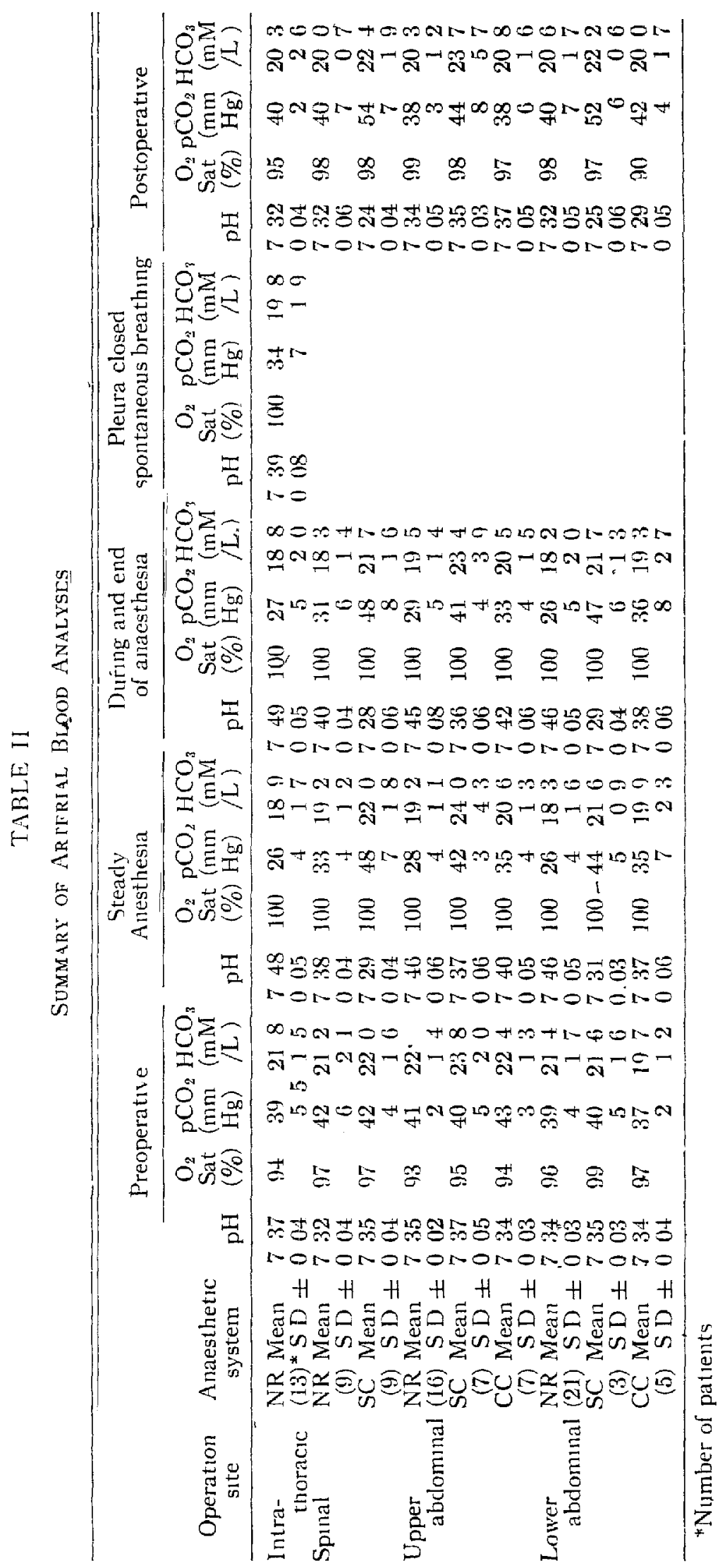


TABLE III

Arterial Blood Analyses on Normal Unmedicated Subjects

\begin{tabular}{|c|c|c|c|c|c|c|c|}
\hline & $\mathrm{pH}$ & $\begin{array}{c}\text { Total } \\
\mathrm{CO}_{2} \\
\text { conitent } \\
(\mathrm{mM} / \mathrm{L})\end{array}$ & $\begin{array}{c}\text { Plasma } \\
\mathrm{CO}_{2} \\
\text { content } \\
(\mathrm{mM} / \mathrm{L})\end{array}$ & $\begin{array}{c}\text { Haematocrit } \\
(\%)\end{array}$ & $\begin{array}{c}\text { Orygen } \\
\text { saturation } \\
(\%) \\
\end{array}$ & $\begin{array}{c}\text { Plasma } \\
\mathrm{HCO}_{3} \\
\text { content } \\
(\mathrm{mM} / \mathrm{L})\end{array}$ & $\begin{array}{c}\mathrm{pCO}_{2} \\
(\mathrm{~mm} \mathrm{Hg})\end{array}$ \\
\hline \multirow[t]{2}{*}{ WS } & 735 & 209 & 252 & 43 & 99 & 239 & 45 \\
\hline & 734 & 211 & 253 & $\frac{13}{43}$ & 97 & 239 & 46 \\
\hline \multirow[t]{2}{*}{$D E$} & $\begin{array}{ll}731 \\
\end{array}$ & 186 & 222 & 4.3 & 95 & 209 & 43 \\
\hline & 732 & 177 & 211 & 41 & & 199 & 40 \\
\hline \multirow[t]{2}{*}{$\mathrm{KV}$} & 734 & 182 & 222 & 43 & 93 & 210 & 42 \\
\hline & 736 & 198 & 238 & 43 & 92 & 226 & 41 \\
\hline \multirow[t]{2}{*}{ GM } & 732 & 185 & 222 & 47 & 96 & 209 & 42 \\
\hline & 732 & 192 & 231 & 49 & 97 & 218 & 44 \\
\hline \multirow[t]{2}{*}{ KYL } & 730 & 181 & 219 & 46 & 100 & 206 & 43 \\
\hline & 732 & 177 & 213 & 45 & 98 & 201 & 40 \\
\hline \multirow[t]{2}{*}{ JK } & 733 & 171 & 19 9 & 38 & 97 & 188 & 37 \\
\hline & 733 & 177 & 208 & 40 & 92 & 197 & 38 \\
\hline \multirow[t]{2}{*}{$V E_{x}$} & 730 & 183 & 213 & 37 & 100 & 201 & 42 \\
\hline & 732 & 179 & 209 & 37 & 100 & 199 & 39 \\
\hline \multirow[t]{2}{*}{ PR } & 734 & 158 & 184 & 36 & 98 & 174 & 33 \\
\hline & 733 & 158 & 183 & 36 & 98 & 173 & 34 \\
\hline \multirow{3}{*}{$\begin{array}{l}\text { Mean } \\
S D= \\
S E=\end{array}$} & 733 & 183 & 217 & 42 & 97 & 206 & 406 \\
\hline & \pm 015 & 15 & 20 & 4 & 25 & 19 & 36 \\
\hline & \pm 005 & 05 & 07 & 15 & 09 & 07 & 13 \\
\hline
\end{tabular}

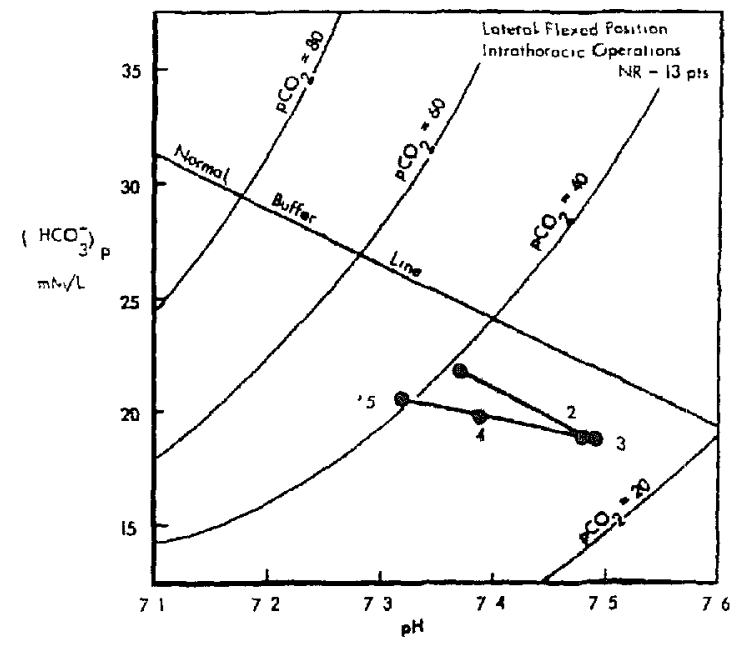

Figure 5 Graph of arterial blood $\mathrm{pH}$ and plasma bicarbonate content

(Mapleson A system) was employed in 29 patients and blood data obtamed from 9 of these revealed that there was a slight but progressive development of respiratory acidosis which persisted after the patient was turned to the supme position (see Figs. 10 and 11). This is the same effect as seen with hand-squeeze ( see Fig. 12). The closed curcle system was employed in only two cases and no blood data were obtaned.

The blood pressure of patients in the prone jackknife position was steady during the anaesthesia, but the pulse pressure was reduced. Most of these patients became moderately hypotensive in the postoperative period, in spite of 


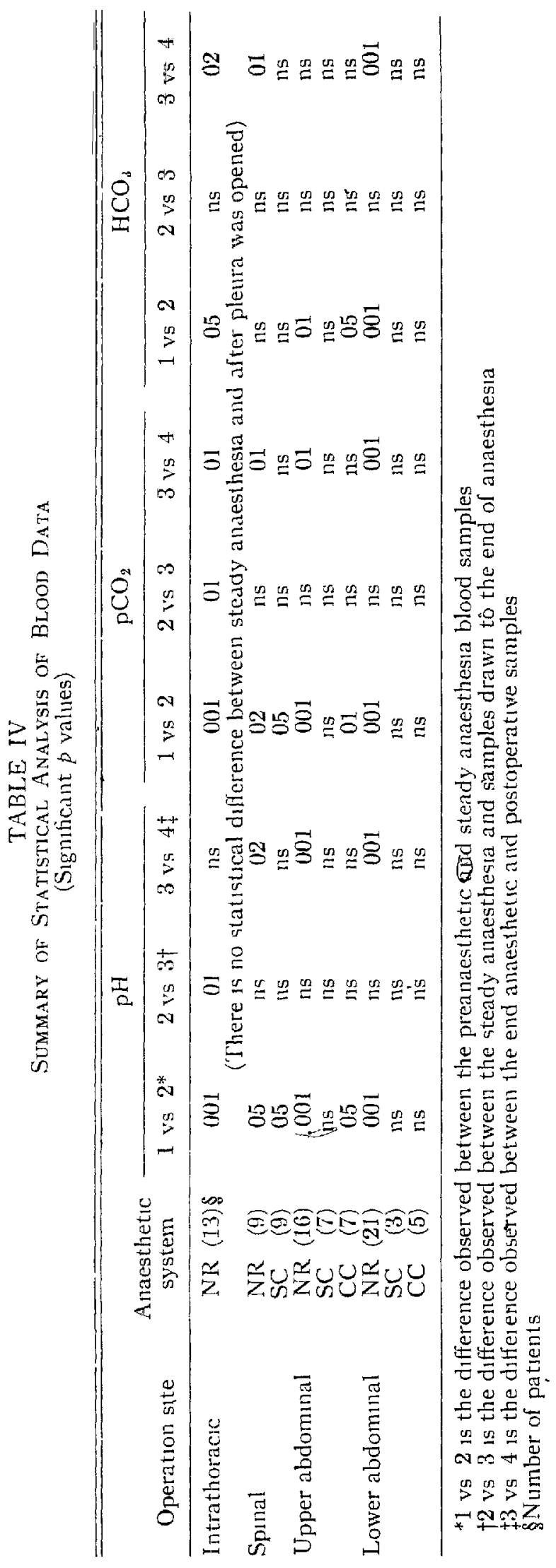




\section{CONTROLLED RESPIRATION STUDY}

Potunt T 9 Hasp 25079 Age 27 Sox M HF, 66" Wr. 126" B 5 A 165 Physicol State Ancesith 2487 Perrce. D 75mg + Scop. 4mg. Anaesthetics Thiop. 425+M 10+NT Build Medium Pult Vert Miertoo Etsten - NR Amplituda 15,-2,0 Phases 111

Blood Proisue Fico, 140/80 Ju ing tricesth Steady (280 minutes) Postop - Steady.

Ventilation Data

Time

Tidal Volume

Rate

Min. Vol. Contral /-_-_-C-General Anaesthesia - - - - -

Arterial Bload Dota

Hbg.

Het.

pH

Totol $\mathrm{CO}_{2}$ content $\mathrm{mM} / \mathrm{L}$ Plasma $\mathrm{CO}_{2}$ content $\mathrm{mM} / \mathrm{L}$ Plasma $\mathrm{HCO}_{3}^{-} \mathrm{mM} / \mathrm{L}$ $\mathrm{PCO}_{2} \mathrm{~mm} \cdot \mathrm{Hg}$.

$\mathrm{O}_{2}$ content Vol. \%

$\mathrm{O}_{2}$ capacity $\mathrm{Vol} . \%$ $\mathrm{PO}_{2} \mathrm{~mm}$. Hg.

$\% \mathrm{O}_{2}$ sofuration

\begin{tabular}{|c|c|c|c|c|c|c|}
\hline 8.45 & 10.15 & 10.45 & 1115 & 12.30 & 1.30 & 2.30 \\
\hline & \multicolumn{6}{|c|}{$550-\ldots 1-550$} \\
\hline 16 & \multicolumn{5}{|c|}{$18-\ldots$} & 24 \\
\hline$S R$ & \multicolumn{5}{|c|}{$\| \mathrm{L}-\ldots-\ldots$} & $S \mathbf{R}$ \\
\hline \multicolumn{2}{|l|}{ Room air } & \multicolumn{5}{|c|}{ Ploura open-..-_..- Pleura elosed Room air } \\
\hline 15.0 & 14.3 & 15.0 & 14.7 & 147 & 14.7 & 14.3 \\
\hline 45 & 43 & 45 & 44 & 44 & 44 & 43 \\
\hline 733 & 7.42 & 7.44 & 7.48 & 744 & 7.39 & 7.39 \\
\hline 19.5 & 16.7 & 15.2 & 14.3 & 14.7 & 172 & 187 \\
\hline 23.5 & 20.3 & 185 & 177 & 18.0 & 209 & 22.4 \\
\hline 225 & 19.4 & 17.7 & 169 & 172 & 19.9 & 21.3 \\
\hline 43 & 31 & 27 & 24 & 26 & 34. & 38 \\
\hline 19.4 & 23.2 & 25.2 & 24.3 & 21.5 & 21.2 & 20.3 \\
\hline 20.1 & 192 & 20.1 & 19.7 & 19.7 & 19.7 & 19.2 \\
\hline 86 & \multicolumn{6}{|c|}{100} \\
\hline 95 & \multicolumn{6}{|c|}{$100-100$} \\
\hline
\end{tabular}

Summary \& Remarks Segmental resections and right upper lobectomy. Lateral flexed position. Anoethesia was maintained with Nitrous oxide, Oxygen (84), and a trace of Trichlorethylene in a Non-rebreathing system. Ventilation wos satisfactory throughout Uneventful course.

Figure 6. Protocol chart of arterial blood data

\section{CONTROLLED RESPIRATION STUDY}

Potient T 25 Hosp. 23935 Age 66 Sox M Hr.65" Wt. 120 B.S.A 1.58 Physicel State 3

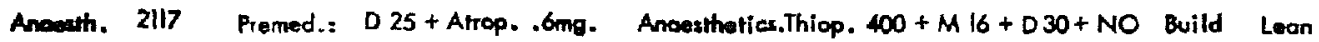

Pulm. Vert Methoc' Etsten - NR Amplitude 15,-2,0 Phoses 1:1.1

Blood Pressu.e. Preop, 110/60

During Anoesth. 110-140/60-90 (180 minutes) Postop,: Uneventful

Ventslation Data

Time

Tidal Volume

Rote

Min. Vol.

Arterial Blood Data

$\mathrm{Hbg}$.

Het.

$\mathrm{pH}$

Total $\mathrm{CO}_{2}$ content $\mathrm{mM} / \mathrm{L}$

Plasmo $\mathrm{CO}_{2}$ content $\mathrm{mM} / \mathrm{L}$

Plasma $\mathrm{HCO}_{3}^{-} \mathrm{mM} / \mathrm{L}$

$\mathrm{PCO}_{2} \mathrm{~mm} . \mathrm{Hg}$.

$\mathrm{O}_{2}$ content Vol.\%

$\mathrm{O}_{2}$ capacty Vol. \%

$\mathrm{PO}_{2} \mathrm{~mm} \cdot \mathrm{Hg}$.

$\% \mathrm{O}_{2}$ soluration

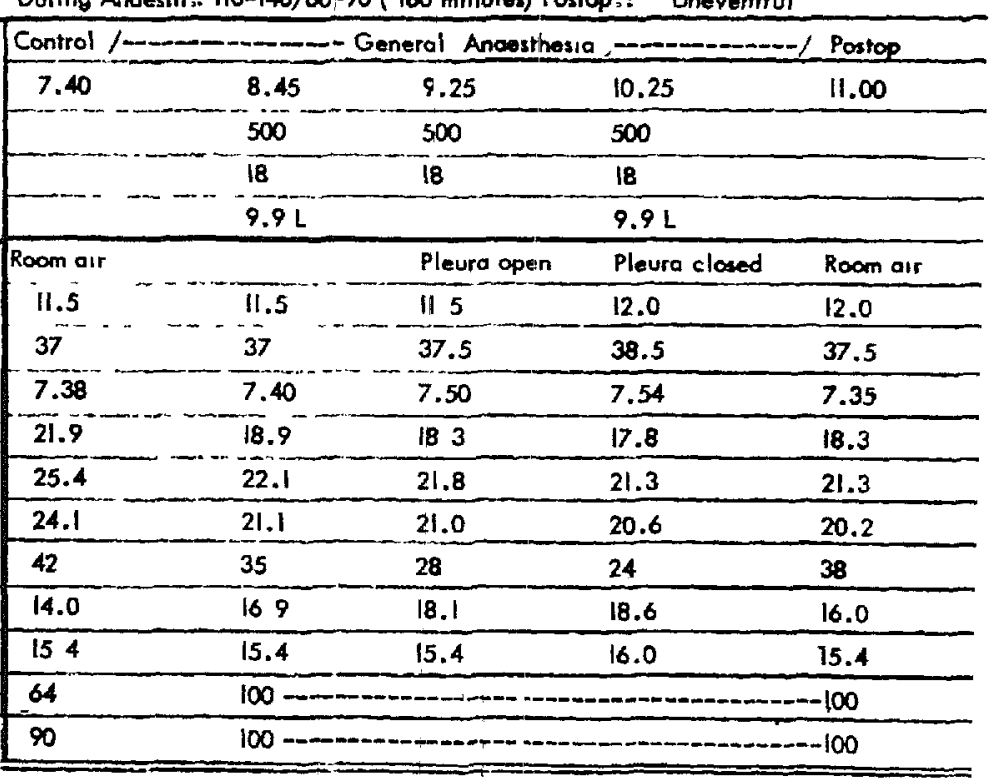

Summary \& Remorks Rught Pneumonectomy and tracheofomy (Bronchogenic carcinomo) Lateral flexed position. Anoesthesia was maintained with Nitrous oxide, Oxygen ( 5:5), and intermittent small amounts of meperidine and dimethyl tubbcurarine intravenously. Note respirqtory alkalosis when pleura was opened. Vital signs were quite steady throughout. Uneventful course postoperatively.

Figure 7 Protocol chart of arterial blood data. 


\section{CONTROLLED RESPIRATION STUDY}

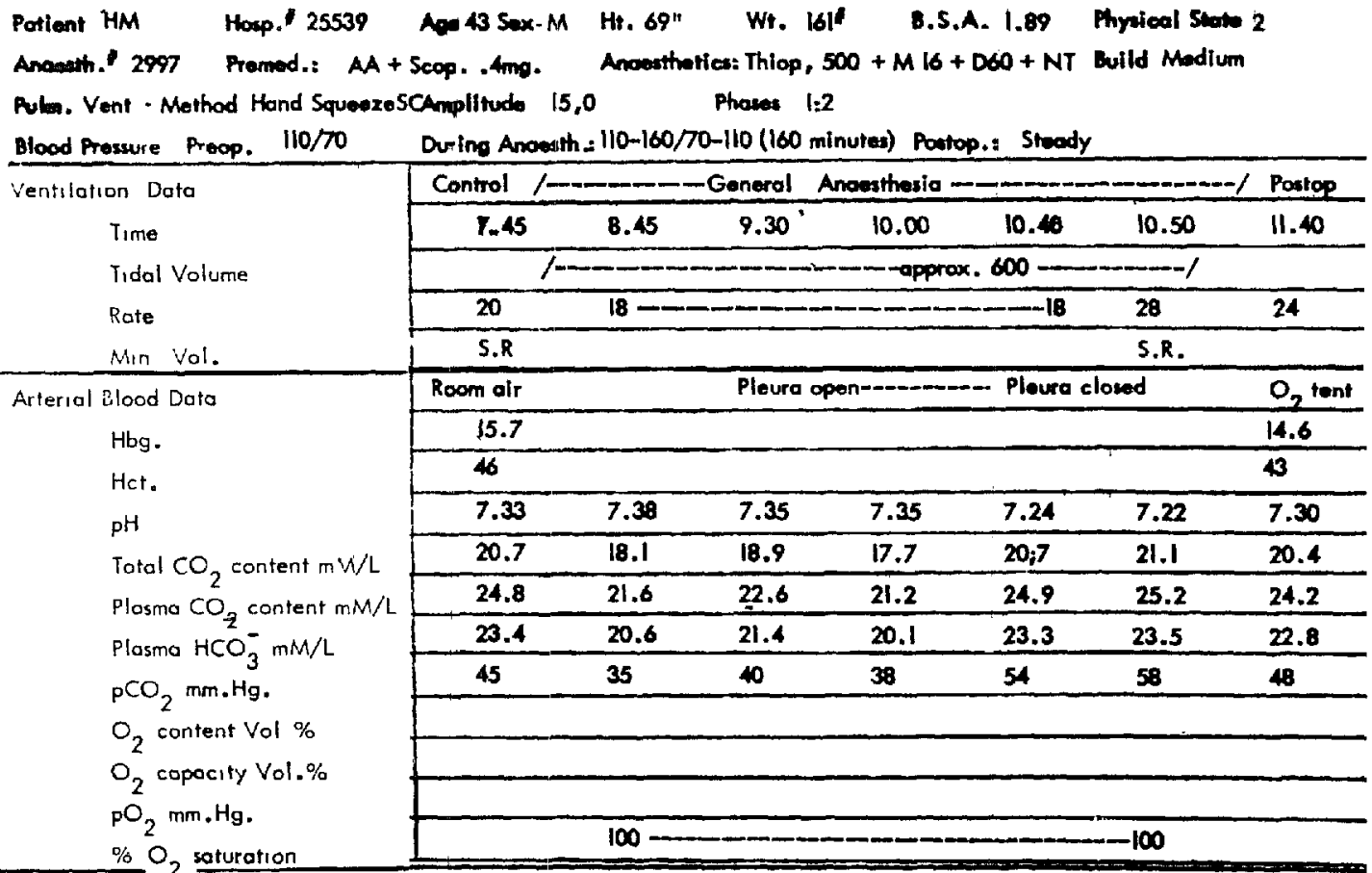

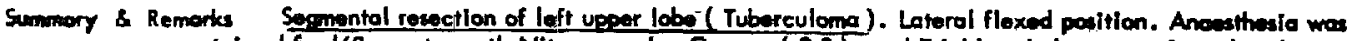
mainiol ned for 160 minuter with Nitrous axidh, Oxyopen (83), and Trichlorathylene in a Semicloed iystem (Mopleson A). Blood presaure was alevated until the ploura was openod, then fell to the preoperative level and rempained zteody into the poitoparative period Except for low grade fever and slight patchy otelectosis in the right lung field (PO $7-9)$ postoperalive course wat ureventful.

Figure 8 Protocol chart of arterial blood data

\section{CONTROLLED RESPIRATION STUDY}

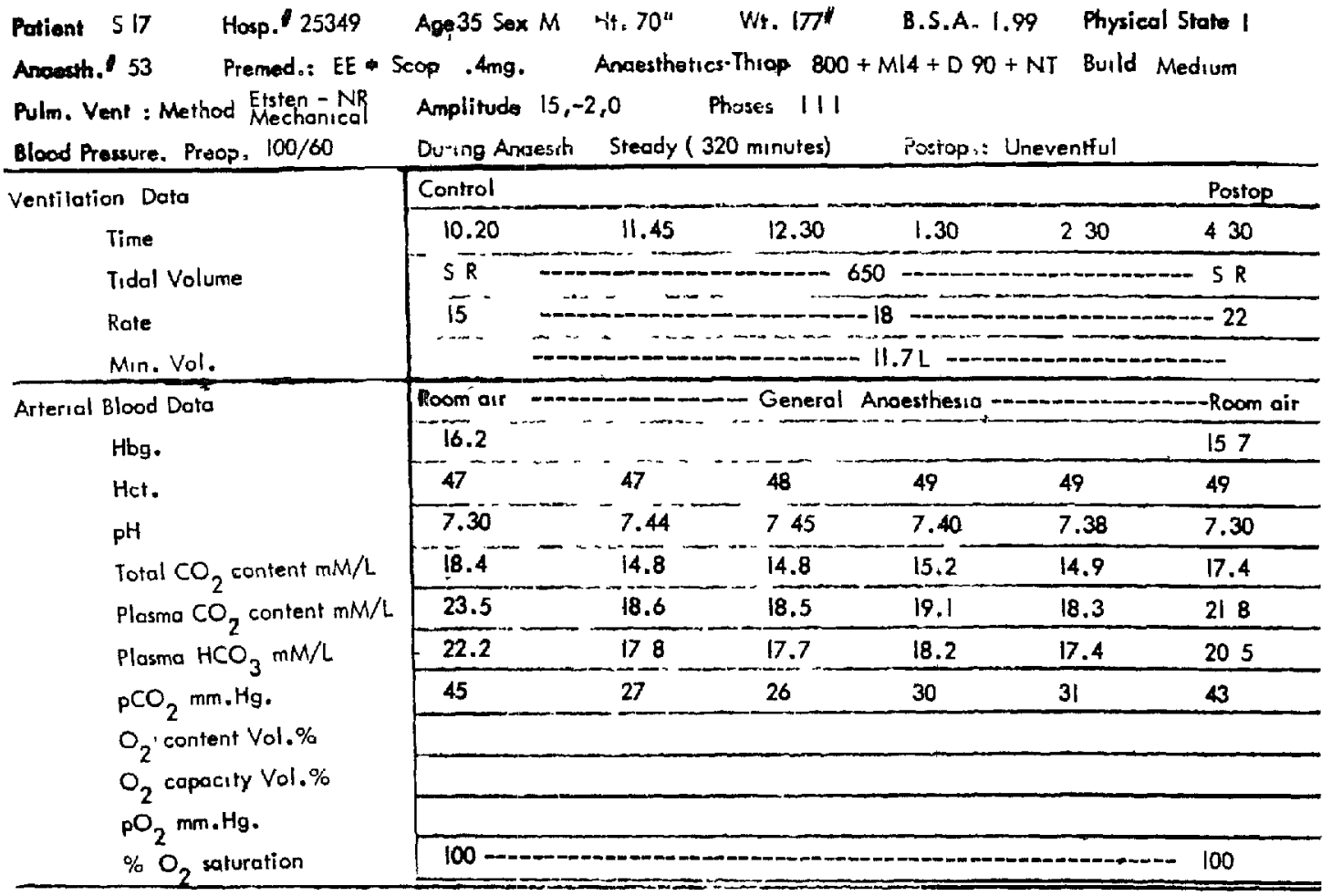

Summory \& Remorks Discoidectomy L4-5. Prone jackknife position. Anoesthesia was maintained for 320 minutes with Nitrous oxide, Oxygen (84), and Trichlorethylene in o Non-rebreathing system. Wneventful course.

Figure 9 Protocol chart of arterial blood data 


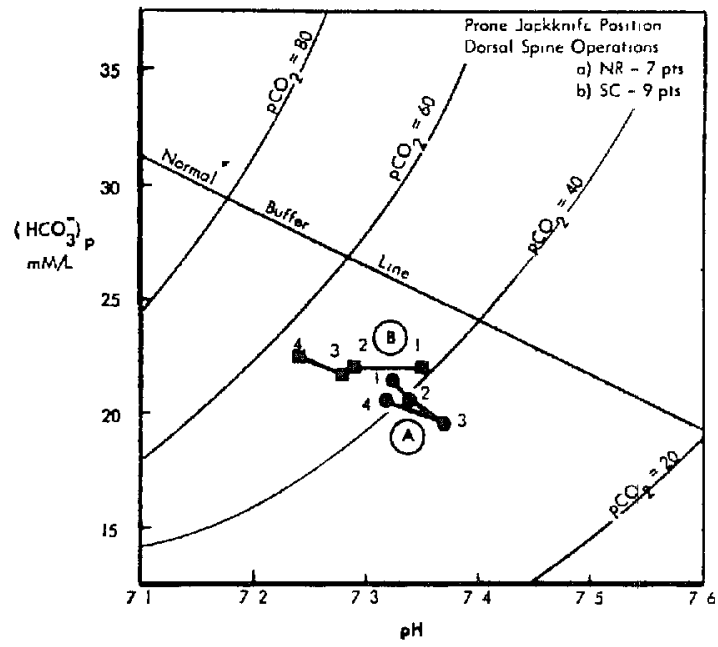

Figure 10 Graph of arterial blood $\mathrm{pH}$ and plasma bicarbonate content

\section{CONTROLLED RESPIRATION STUDY}

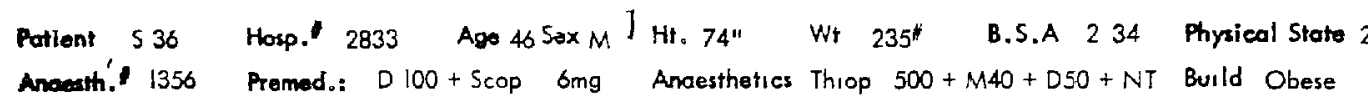

Pulm. Vent Mathod Etsten - SC Amplitucle 15,-2,0 Phases ILI

Blood Pressure. Preop 124/84 Jüing Aricesit. Stecdy (270 minutes) Postop. Uneventful

\begin{tabular}{|c|c|c|c|}
\hline Ventilation Data" & Control --..-- General Anaesthesia - & - & Postop \\
\hline Time & 9.00 & 1110 & 110 \\
\hline Tidal Valume & $5 R=5$ & $750 \cdots$ & $S R$ \\
\hline Rate & 18 & 18 & \\
\hline Min Vol & $135 \mathrm{~L}$ & $135 \mathrm{~L}$ & \\
\hline Arterial Blood Dato & Roöm air & & Room air \\
\hline $\mathrm{Hbg}$. & 146 & & 156 \\
\hline Hct. & 485 & 50 & 48 \\
\hline $\mathrm{pH}$ & 735 & 719 & 722 \\
\hline Total $\mathrm{CO}_{2}$ content $\mathrm{mM} / \mathrm{L}$ & 186 & 199 & 184 \\
\hline Plasma $\mathrm{CO}_{2}$ content $\mathrm{mM} / \mathrm{L}$ & 224 & 238 & 221 \\
\hline Plasma $\mathrm{HCO}_{3}^{-} \mathrm{mM} / \mathrm{L}$ & 200 & 22.0 & 205 \\
\hline $\mathrm{PCO}_{2} \mathrm{~mm} \cdot \mathrm{Hg}$ & 39 & 60 & 52 \\
\hline $\mathrm{O}_{2}$ content Yol. $\%$ & 24.8 & 245 & 227 \\
\hline $\mathrm{O}_{2}$ copacity Vol. $\%$ & 202 & 20.2 & 202 \\
\hline $\mathrm{PO}_{2} \mathrm{~mm} \cdot \mathrm{Hg}$ & $100-1-\ldots$ & \multicolumn{2}{|c|}{$100-100$} \\
\hline$\% \mathrm{O}_{2}$ saturation & $100-1-1-1$ & \multicolumn{2}{|c|}{$100-100$} \\
\hline
\end{tabular}

Fummary \& Remorks Discoidectomy L5 - SI, \& Spinal fusion. Prone jackknife plosition Anaesthesia was maintained for 270 minutes with Nitrous oxide, Oxygen (73), and Trichlorethylene $V_{1}$ tal signs were steody throughout in spite of progressive respiratory acidosis. This acidosis is a characteristic occurrence when the semiclosed system of the Mogilf type (Mapleson A) is employed for patients in the prone position even when pulmonary ventilation appears to be adequate.

Ficune 11 Protocol chart of arterial blood data 
Pationt LW Hosp. 21313 age 42 Sex F Hi. 65" Wr. 136" B S.A. L.67 Physical Statell! Anoesth. 1523 Premed.: D 50mg. + Atrop. .6mgtice. "ilehiss: Thiop.400+M14 D 20+NT Build Medium Pulm. Vent. Method Hand Squeeze Amositucic 15,0 Phijes 12

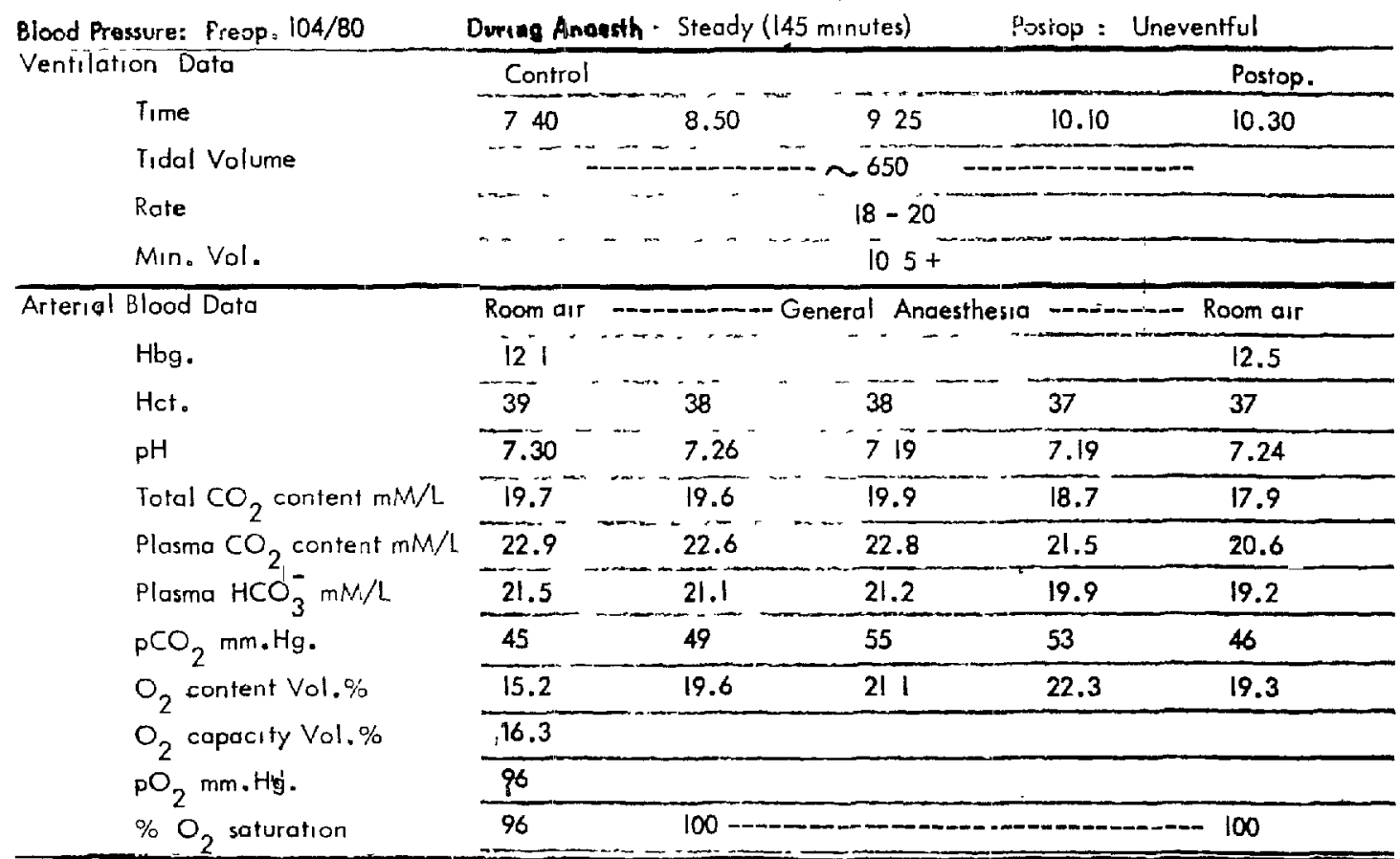

Summory \& Remarks Discoidectomy. Prone jackknife position. Anaesthesia was maintained for 145 minutes with Nitrous oxide, Oxyigen ( 63 ), and Trichlorethylene in a Semiclosed system without $\mathrm{CO}_{2}$ absorption (Mapleson A). Note progressive respiratory acidosis. Vital signs'remained stable. ${ }^{2}$

Frgure 12 Protocol chart of arterial blood data

bandaging the legs This was most evident when the semi-closed system was employed However, the blood was fully saturated with oxygen and there was no significant accumulation of fixed acids. The latter indicates that there is no metabolic disturbance due to trichlorethylene, even when a shght respiratory acidosis develops.

Upper abdominal operations. During the course of 30 of the 132 operations in the supine horizontal position, 155 arterial blood samples were analysed. The non-rebreathing system was employed in 58 patients Blood data obtained from 16 of these revealed that pulmonary ventılation was always adequate (see Figs. 13, 14, and 15). Moderate respiratory acidosis was observed postoperatively in one patient-an obese man who had a tight abdominal binder applied following repair of a large ventral hernia. This evidently splinted the diaphragm sufficiently to impede ventilation. The seml-closed system was employed in 26 patients. Blood data obtained from 7 of these revealed that pulmonary ventilation was adequate in each case (see Figs. 16 and 17). The closed circle system was employed in 48 patients and blood data obtained from 7 of these also revealed that pulmonary ventilation was adequate in every case (see Figs. 17 and 18). 


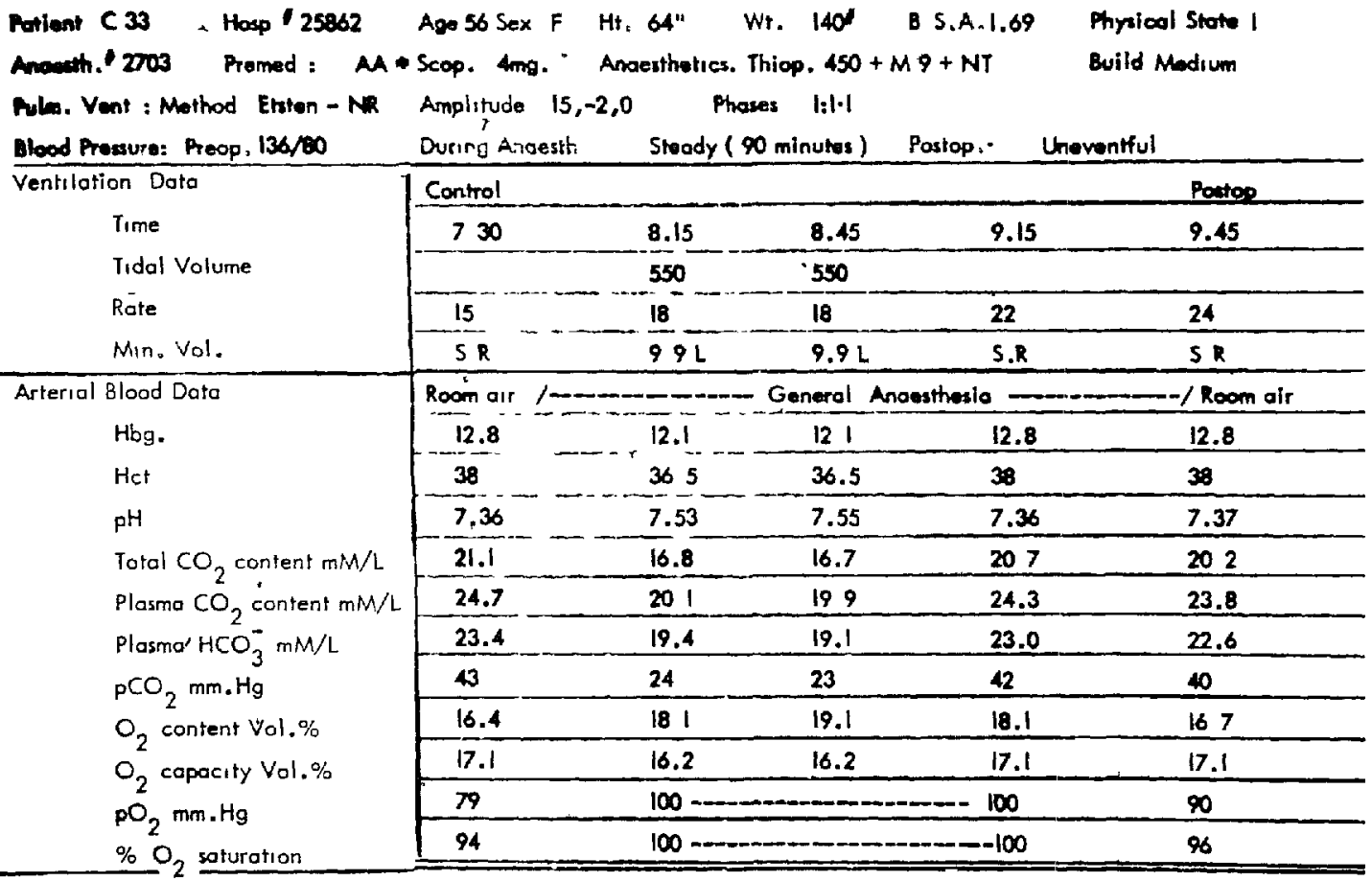

Summory \& Remorks Cholecystectomy and appendectomy Supine horizontal position Anaesthesia was maintained with Nitrous oxido, Oxygen (7.3), and a trace of Trichlorethylene, in a Non - rebreathing system. Mild respiratory alkalosis Juring anaesthesia. Uneventful course.

FIGURE 13 Protocol chart of arterial blood data

$$
\text { CONTROLLED RESPIRATION STUDY }
$$

Pationt G 24 Hosp 23672 Ago 65 Sex M Hi 6l" vit $107^{\prime \prime}$ B SA 145 Ptysical State 2 Ancousth. 2512 Premed; D 50mg + Atrop .6mg nnactiheriss Thiop. $450+M 13+N T$ Build Lean Pulm. Vent Method Eisten - NR Ainplitude 13,-2,0 Pheses 111

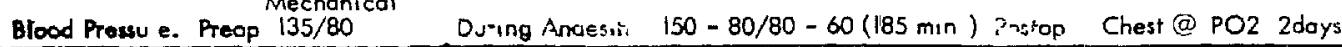

\begin{tabular}{|c|c|c|c|c|c|}
\hline \multirow{5}{*}{$\begin{array}{l}\text { Ventilatian Dato } \\
\text { Time } \\
\text { Tidal Volume } \\
\text { Rate } \\
\text { Min Vol. } \\
\end{array}$} & \multicolumn{5}{|c|}{ Control } \\
\hline & 938 & $1030 \ldots 11.15$ & 1215 & 100 & 130 \\
\hline & $S R$ & 600 & -1 & -600 & $5 R$ \\
\hline & 16 & i8 $-1-2=-1$ & $=-1$ & & 22 \\
\hline & \multicolumn{5}{|c|}{$108 \mathrm{~L}$} \\
\hline \multirow{12}{*}{$\begin{array}{l}\text { Total } \mathrm{CO}_{2} \text { content mi/L } \\
\text { Plasma } \mathrm{CO}_{2} \text { content mM/L } \\
\text { Plasma } \mathrm{HCO}_{3}^{-} \mathrm{mM} / \mathrm{L} \\
\mathrm{pCO}_{2} \mathrm{~mm} \cdot \mathrm{Hg} \\
\mathrm{O}_{2} \text { content } \mathrm{Vol} . \% \\
\mathrm{O}_{2} \text { cápocity } \mathrm{Val.} \% \\
\mathrm{PO}_{2} \text { mm. Hg } \\
\% \mathrm{O}_{2} \text { saturation }\end{array}$} & Room air & $---\infty-G$ & Anaesth & -1 & Room air \\
\hline & 11.6 & & & & 123 \\
\hline & 35 & 34 & 36 & 36 & 36 \\
\hline & 736 & 7.54 & 7.45 & 7.45 & 7.31 \\
\hline & 20.7 & 17.3 & 17.0 & 17.3 & 189 \\
\hline & 23.9 & 20.4 & 20.4 & 21.7 & 22.8 \\
\hline & 22.7 & 18.6 & 19.6 & 20.8 & 216 \\
\hline & 41 & 22 & 28 & 29 & 42 \\
\hline & 146 & 20.6 & 19.8 & 19.1 & 167 \\
\hline & 15.6 & & & & \\
\hline & & & & & \\
\hline & 92 & $100 \ldots$ & -- & $-\infty$ & 100 \\
\hline
\end{tabular}

Funnory \& Remorks Subtotal Gastrectomy. Supine horizontal position. Anaesthesia was mointained for 185 minutes with Nitrous oxide, Oxygen ( 8.3 ), and a troce of Trichlorethylene in a Non - rebreathing system. Vital signs remained steady following moderate decrease of blood pressure during induetion. Note that moderate respiratory alkalosis developed while the patient was ventilated mochanically. Anaesthetic course was unevenfful otherwise. Fever and chest rales oceurred on PO2, and lasted 2 days.

Figure 14. Protocol chart of arterial blood data. 


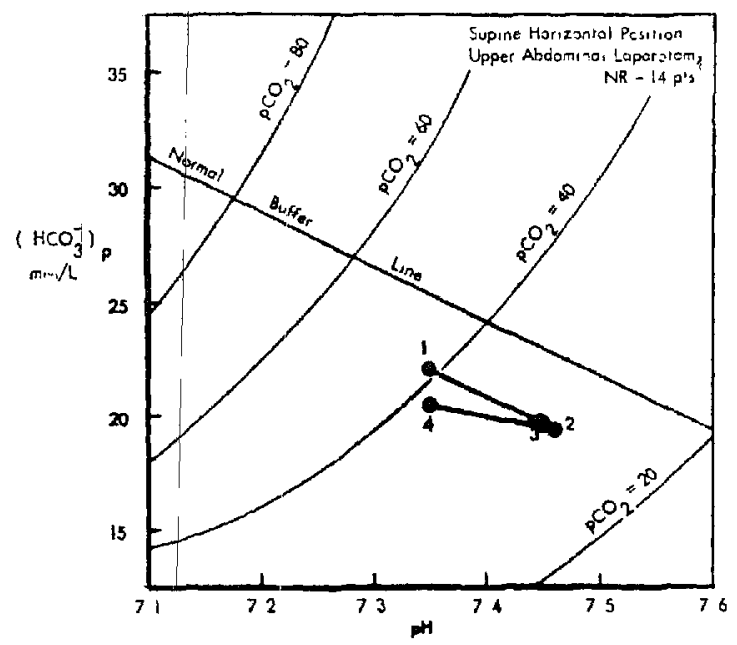

Frcure 15 Graph of arterial blood $\mathrm{pH}$ and plasma bicarbonate content

\section{CONTRQLLED RESPIRATION STUDY}

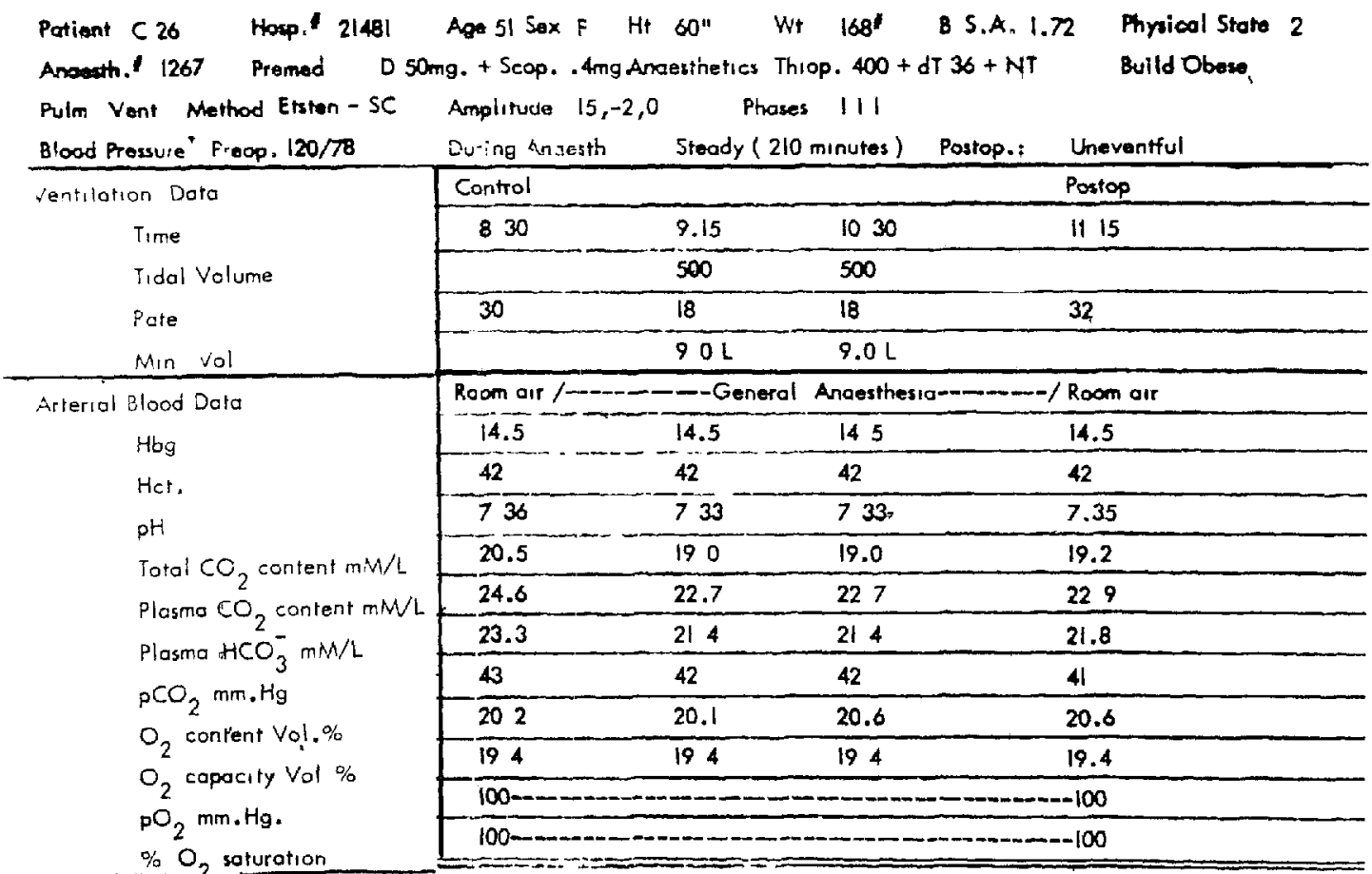

Summory \& Remorks Cholecystectomy, vagotomy, pyloroplasty and appendectomy Anaesthesia was mointained with Nitrous oxido, Oxygen (73), and a troce of Trichlorethylene, in a Semiclosed system (Mopleson A). No alteration in $\mathrm{PCO}_{2}$. Uneventful course

Figure 16 Protocol chant of arterial blood data 


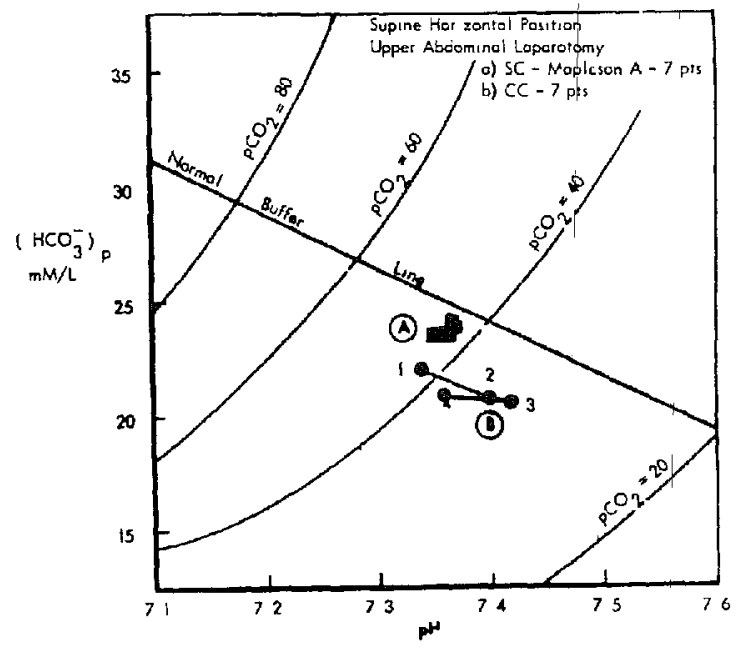

Figure 17 Graph of arterial blood $\mathrm{pH}$ and plasma bicarbonate content

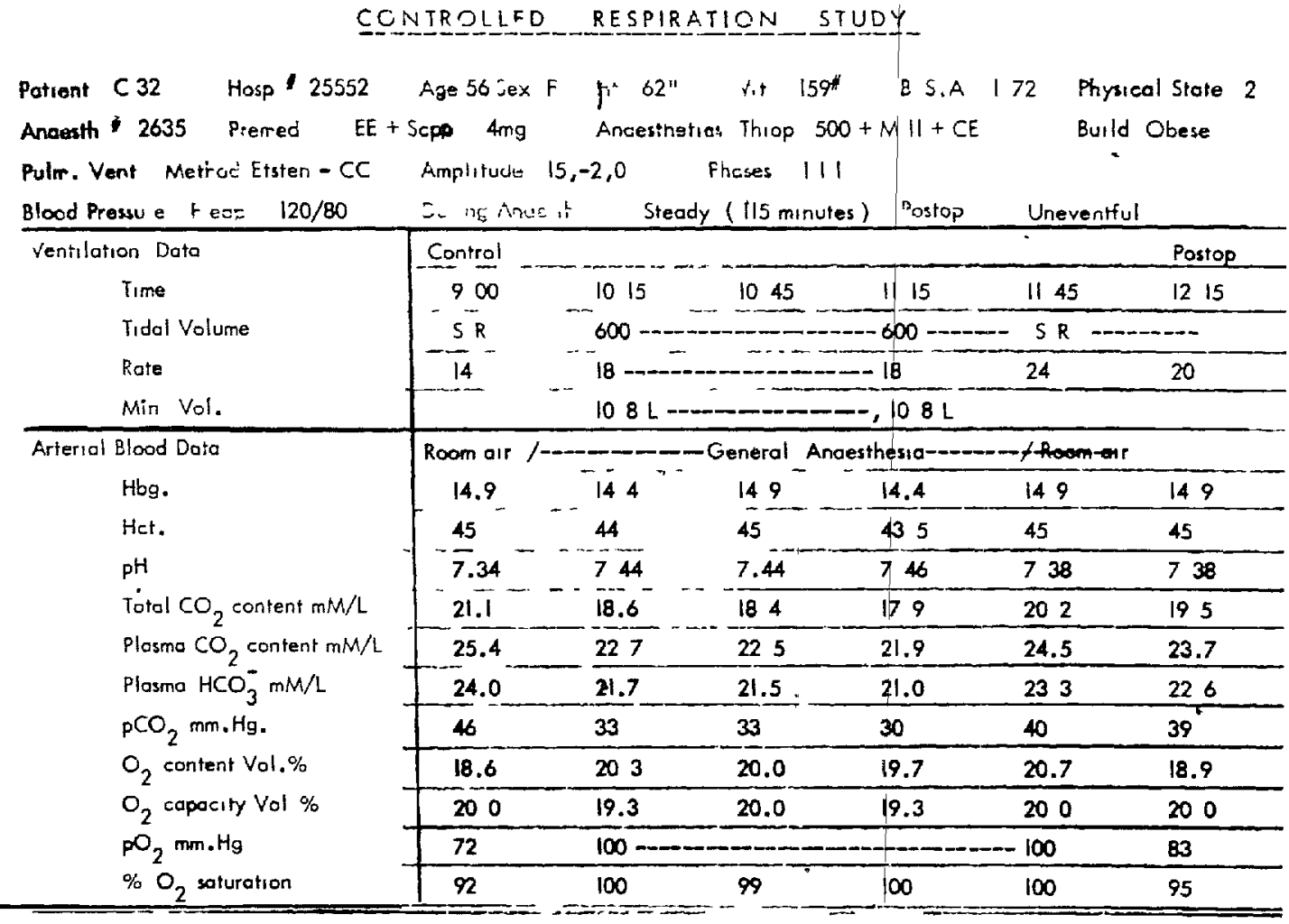

5ummory \& Rerrarks Cholecystectomy. Supine horizontal posiyion Anaesthesia was maintained for 115 minutes with Cyclopropane and a trace of Ether in a Closed Circle system with $\mathrm{CO}_{2}$ absorption. Ventilation was sctisfactory. Uneventful course.

Figure 18 Protocol chart of artenal blood data. 
Lower abdominal operations . Durmg 29 of the 115 operations in the head low or Trendelenburg position, 143 arterial blood samples were analysed. The nonrebreathing system was employed in 51 patients Blood data obtained in 21 of these revealed that pulmonary ventilation was adequate in every patient (see Figs 19, 20, and 21) The sem1-closed system was employed in 14 patients Blood data obtained from 3 of these revealed that there was no statistically significant change, but a slight progressive trend toward respiratory acidosis was evident (see Fig 22) The closed crrcle system was employed in 50 patients Blood data obtaned from 5 of these revealed that pulmonary ventilation was adequate in each case (see Figs 22, 23, and 24).

The 112 patients on whom automatic control of pulmonary ventilation was used were selected among the four groups described above Its use was limited manly to those patients who were poor risks from an anaesthetic and surgical point of view and/or who were extremely obese or emphysematous, and when a "third hand" would be a considerable advantage in the management of the anaesthetic In no procedure was it found necessary to discontmue the use of the motor owing to mechamical difficulty or to difficulty in providing the desirable pressure amplitude. In a few patients it was thought desirable to have a longer expiration phase at atmospheric pressure, but this could not be adjusted However, no apparent difficulty developed when a compromise adjustment was

$$
\text { CONIROLLED RESPIRATION STUDY }
$$

\begin{tabular}{|c|c|c|c|c|c|c|c|}
\hline \multicolumn{2}{|c|}{ Anoesth. F 2235 Promed : D $50 \mathrm{mg}$} & $\begin{array}{l}\text { p. } 9998 \\
\text { med : D } 50 \mathrm{mg}\end{array}$ & $\begin{array}{l}\text { Age } 49 \text { Sex } F \\
+ \text { Atrop } 4 \mathrm{mg}\end{array}$ & \multicolumn{2}{|c|}{ Anoesthetics: Thiop. $425+M 12+N I$} & \multicolumn{2}{|c|}{$\begin{array}{l}\text { Physical State } 3 \\
\text { Build Medium }\end{array}$} \\
\hline Pulm. V & Jont. Method & Etsten - NR & Amplitude & Phases 111 & & \\
\hline \multicolumn{2}{|c|}{ Blood Prossure- Preop } & $110 / 60$ & During Angessh. & Steady ( 160 minutes ) & \multicolumn{2}{|c|}{ Postíp: : Uneventful } & \\
\hline \multicolumn{3}{|c|}{ Ventslation Dota } & Control & & & & - Postop \\
\hline \multicolumn{3}{|c|}{ Time } & 745 & 855 & 9.55 & 10.35 & 11.25 \\
\hline \multicolumn{3}{|c|}{ Tidal Volume } & $5 \mathrm{R}$ & \multicolumn{3}{|c|}{$550-2-550$} & SR \\
\hline \multicolumn{3}{|c|}{ Rate } & 20 & \multicolumn{3}{|c|}{$18=-18$} & 24 \\
\hline \multicolumn{3}{|c|}{ Min. Vol. } & \multicolumn{5}{|c|}{$9.9 \mathrm{~L}-\cdots, 9 \mathrm{~L}$} \\
\hline \multicolumn{3}{|c|}{ Arterial Blood Data } & Room air $/-$ & $\ldots-G$ General An & gesthesia--.--.... & -1 & Room air \\
\hline \multicolumn{3}{|c|}{ Hbg. } & 9.9 & -105 & 10.8 & 10.8 & 108 \\
\hline \multicolumn{3}{|c|}{ Hct } & 31 & 33 & 34 & 34 & 34 \\
\hline \multicolumn{3}{|c|}{$\mathrm{pH}$} & 7.36 & 7.46 & 7.52 & 752 & 7.34 \\
\hline \multicolumn{3}{|c|}{ Total $\mathrm{CO}_{2}$ content $\mathrm{mM} / \mathrm{L}$} & 19.3 & 17.3 & 16.2 . & 16.6 & 18.8 \\
\hline \multicolumn{3}{|c|}{ Plasmo $\mathrm{CO}_{2}$ cantent $\mathrm{mM} / \mathrm{L}$} & 22.0 & 20.0 & 18.9 & 19.4 & 21.5 \\
\hline \multicolumn{3}{|c|}{ Plasmo $\mathrm{HCO}_{3}^{-} \mathrm{mM} / \mathrm{L}$} & 20.9 & 19.2 & 18.2 & 18.7 & 20.3 \\
\hline \multicolumn{3}{|c|}{$\mathrm{pCO}_{2} \mathrm{~mm} \cdot \mathrm{Hg}$} & 38 & 28 & 23 & 24 & 39 \\
\hline \multicolumn{3}{|c|}{$\mathrm{O}_{2}$ content Vol.\% } & 13.4 & 16.9 & 19.2 & 16.7 & 165 \\
\hline \multicolumn{3}{|c|}{$\mathrm{O}_{2}$ capacity Vol. $\%$} & 13.2 & 132 & 14,5 & 14.5 & 14.5 \\
\hline \multicolumn{8}{|c|}{$\mathrm{PO}_{2} \mathrm{~mm} \cdot \mathrm{Hg}$} \\
\hline \multicolumn{3}{|c|}{$\% \mathrm{O}_{2}$ saturation. } & 99 & $100 \ldots$ & $-\infty-$ & $-\infty$ & 100 \\
\hline
\end{tabular}

Summory \& Romerks Total hysterectamy. Head low $15^{\circ}$ position.. Anaesthesia was maintained for 155 minutes with Nitrous oxide, Oxygen (8:3), arid a trace of Trichlorethylene in a Non-rebreathing system. Vitol signs stable throughout.

Figune 19 Protocol chart of arterial blood data 
made. During many of the anaesthetics, the employment of this device greatly facilitated the management of the patient, and in a few cases it obviously avoided the development of a harassing situation

\section{CONTROLLED RESPIRATION STUDY}

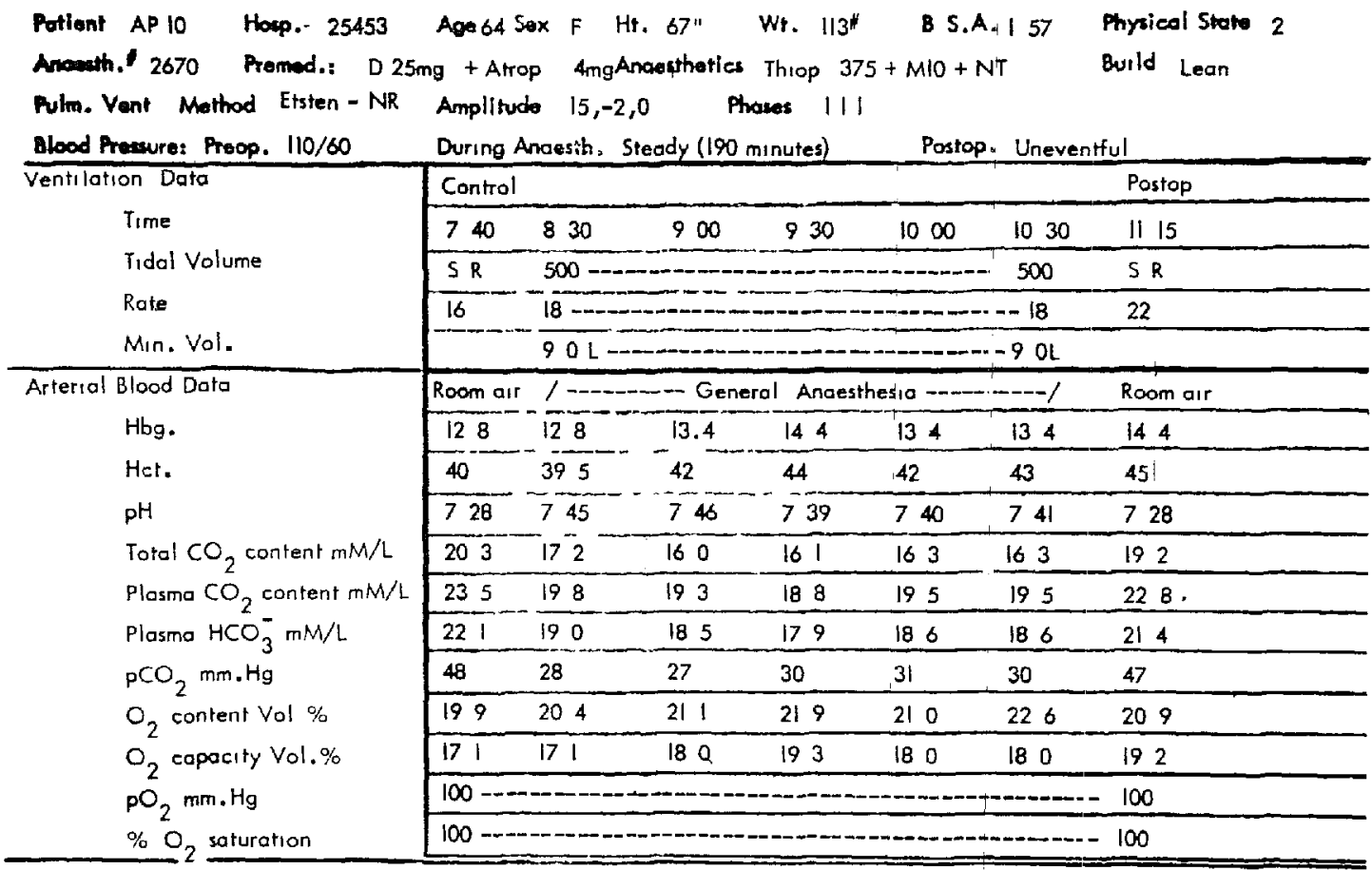

Sammery \& Remarks Abdaminal perineal resection Lithotomy position with $10^{\circ}$ head low Angesthesia was maintained with Nitrous oxide (9L), Oxygen (3L), and a trace of Trichlorethylene, in a non-rebreathing system Ventilation was satisfactory throughout There was a mild respiratory acidosis preaperatıvely, which returnied of ter artificial respiration was discontinued

Ficure 20 Protocol chart of arterial blood data

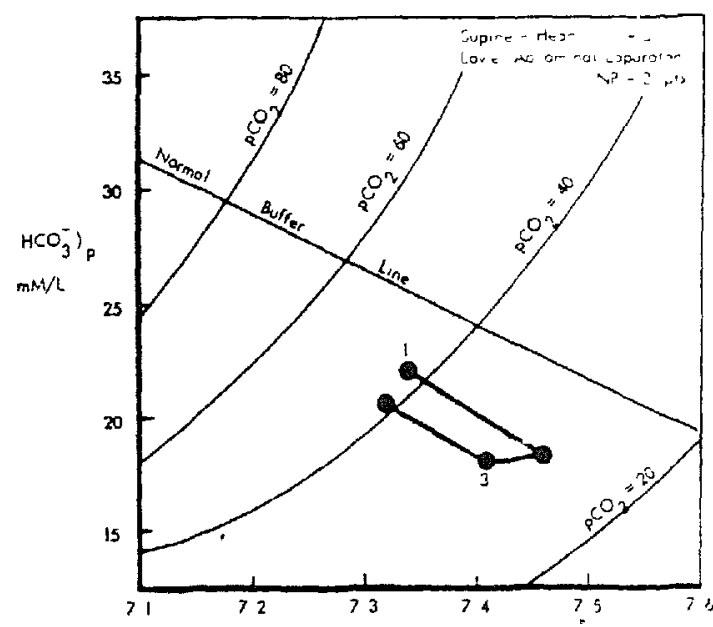

Figure 21. Graph of arterial blood $\mathrm{pH}$ and plasma bicarbonate content

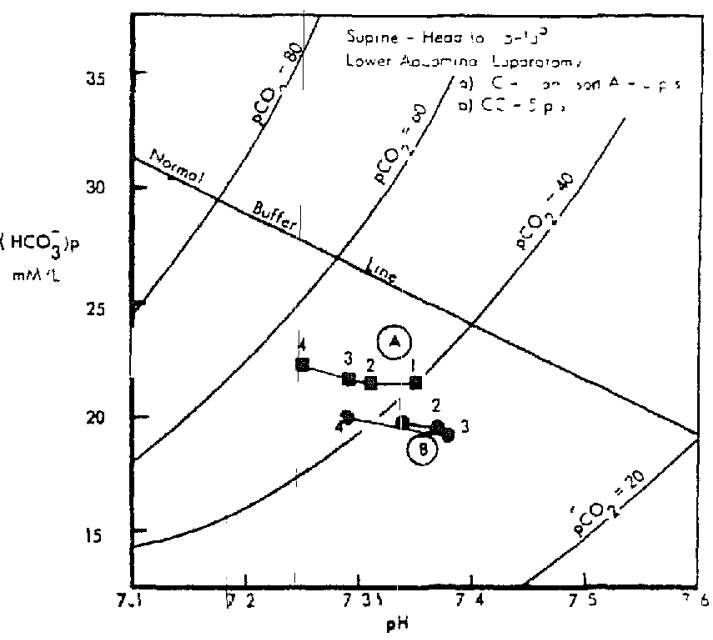

Figure 22 Graph of arterial blood pH and plasma bicarbonate content 


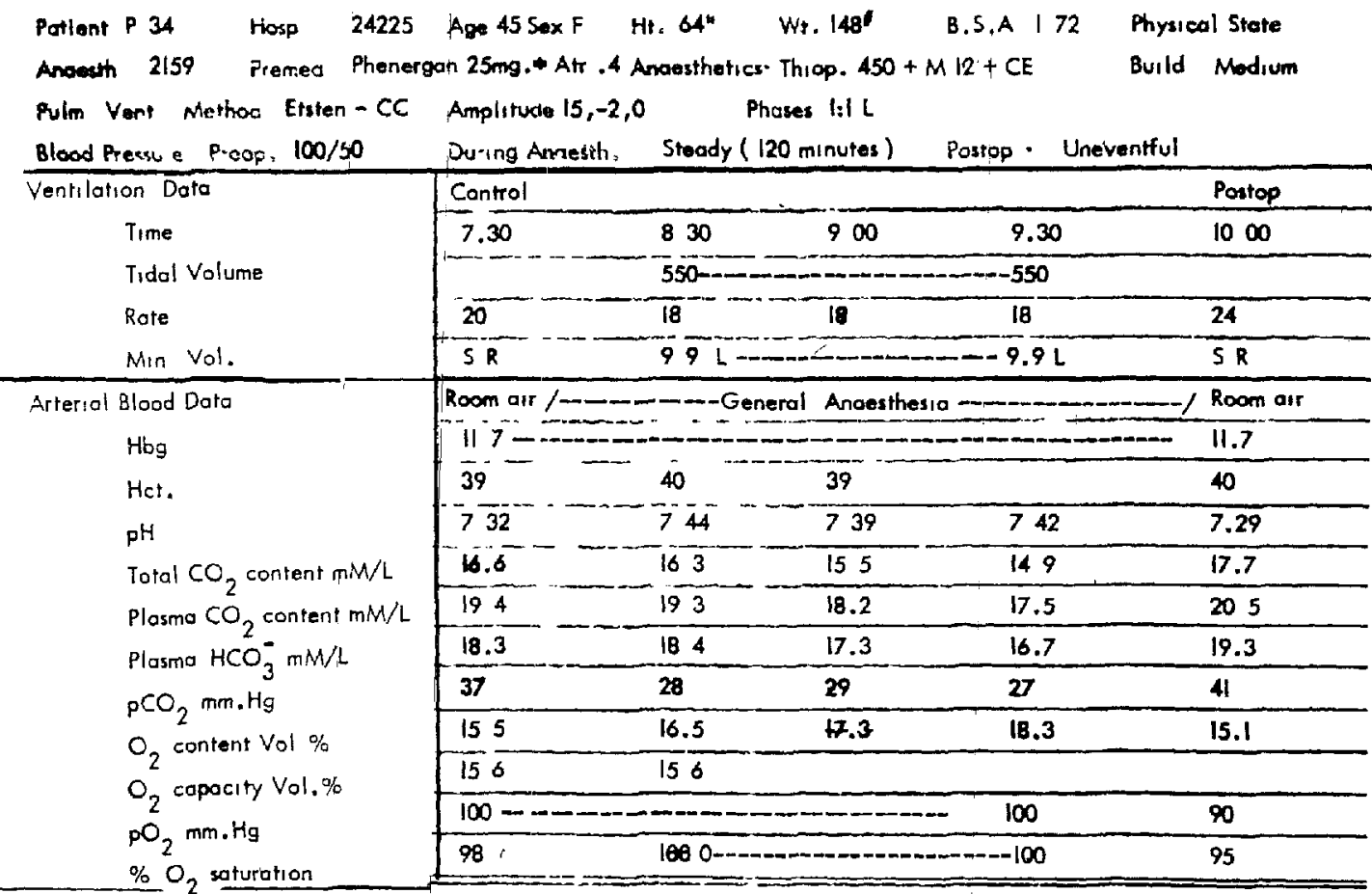

Summary \& Remaiks Total Hysterectomy Head low $15^{\circ}$ (Trendelenberg) position Anaesthesia was maintained for 115 minutes with Cyclopropone and a trace of Ether in a Elosed Circle system with $\mathrm{CO}_{2}$ absorption Uneventful postoperative clourse

Figune 23 Protocol chart of arterial blood data

\title{
CONTROLLED RESPIRATION STUDY
}

\begin{abstract}
Patient AP7 Howp.- 25422 Age62 Sax M ht. 66" Wt. $130^{\circ}$ B.S.A. I.66 Phyticol State 3
\end{abstract}
Anoetth. 2602 Premed.; AA + Scop 4ng Ancesthotics Thiop 450+MB+CE Build Modium

Pulm. Vont Mathod Emsten-CC Amolitude 15,-2,0 Phases 111

Blocd Preasure. Preop $110 / 70$ Durng Arraestit, Elevared while legs ralsed Postop,: Steady

\begin{tabular}{|c|c|c|c|c|c|c|c|c|c|}
\hline \multirow{5}{*}{$\begin{array}{l}\text { Ventilation Data } \\
\text { Time } \\
\text { Tidal Volume } \\
\text { Rate } \\
\text { Min Vol }\end{array}$} & \multirow{3}{*}{\multicolumn{7}{|c|}{\begin{tabular}{|lllllllllll}
7 & 40 & 8 & 35 & 9.05 & 9 & 35 & 10.05 & 10.35 & 11.05 & 11.35 \\
\end{tabular}}} & \multirow{2}{*}{\multicolumn{2}{|c|}{$\frac{\text { Postop }}{12.40}$}} \\
\hline & & & & & & & & & \\
\hline & $S R$ & & & & & & $600=-600$ & \multicolumn{2}{|c|}{ S R - - - } \\
\hline & 14 & \multicolumn{8}{|c|}{$\overrightarrow{8}-18-20$} \\
\hline & & \multicolumn{8}{|c|}{$108 \mathrm{~L} \cdots-10.8$} \\
\hline \multicolumn{10}{|c|}{ Room air / / - Room air } \\
\hline Hbg. & 130 & 135 & 135 & 14.5 & 141 & 135 & 115 & 12.0 & 115 \\
\hline $\mathrm{Het}$ & 40 & 41 & 415 & 45 & 43.5 & 41 & 355 & 37 & 35.5 \\
\hline $\mathrm{pH}$ & 734 & 743 & 744 & 745 & 744 & 7.44 & 7.44 & 726 & 7.28 \\
\hline \multirow{3}{*}{$\begin{array}{l}\text { Total } \mathrm{CO}_{2} \text { content } \mathrm{mM} / \mathrm{L} \\
\text { Plasma } \mathrm{CO}_{2} \text { content } \mathrm{mM} / \mathrm{L}\end{array}$} & 17.5 & 14.9 & 15.4 & 144 & 14.4 & 154 & 143 & 17.4 & 164 \\
\hline & 20.6 & 17.9 & 185 & 17.7 & 175 & 185 & 168 & 200 & 18.8 \\
\hline & 19.5 & 17.1 & 177 & 170 & 17.3 & 17,7 & 161 & 18.7 & 176 \\
\hline \multirow{5}{*}{$\begin{array}{l}\mathrm{pCO}_{2} \mathrm{~mm} . \mathrm{Hg} . \\
\mathrm{O}_{2} \text { content Vol.\% } \\
\mathrm{O}_{2} \text { capacity Vol.\%" } \\
\mathrm{pO}_{2} \mathrm{~mm} . \mathrm{Hg} \\
\% \mathrm{O}_{2} \text { saturation }\end{array}$} & 37 & 27 & 26 & 25 & 26 & 27 & 24 & 43 & 39 \\
\hline & 17.3 & 20.9 & 199 & 20.1 & 20.8 & 19.6 & 18.1 & 18.0 & 14.1 \\
\hline & 17.4 & 18.1 & 181 & 196 & $18.9 \quad 18.9$ & 18.1 & 15.4 & 16.1 & 15.4 \\
\hline & \multicolumn{9}{|c|}{100090} \\
\hline & \multicolumn{9}{|c|}{$98 \quad 100-1$} \\
\hline
\end{tabular}

Summory \& Romarks Abdominal perineal resection Lithotomy position with $10^{\circ}$ hood low. Anassthesia mas maintained with 10 to $15 \%$ Cyclopropane, and a trace of Ether in a closed circle $\mathrm{CO}_{2}$ obsorption system. Severo bleading and pelvic traction during the late part of the operation caused a marked fallin blood pressure, which stabilized after atropine, rapid blood transfusion and a vacoprescor. Postanaesthetic course wat uneventful. There mas a mild metabolic acidosis in the immediate postoperative period.

Figure 24 Protocal chart of artenal blood dat 


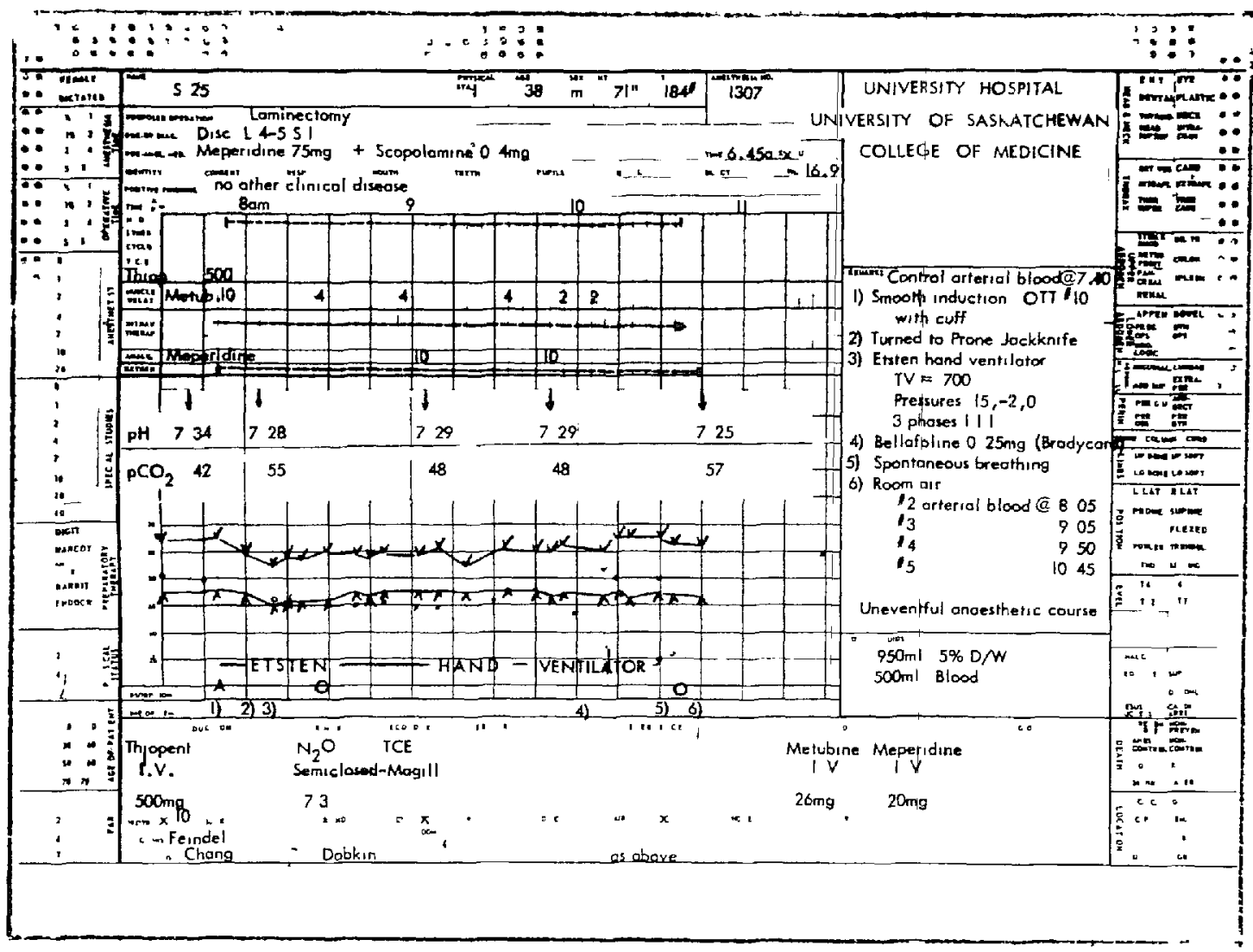

Figure 25 Anaesthetx chart

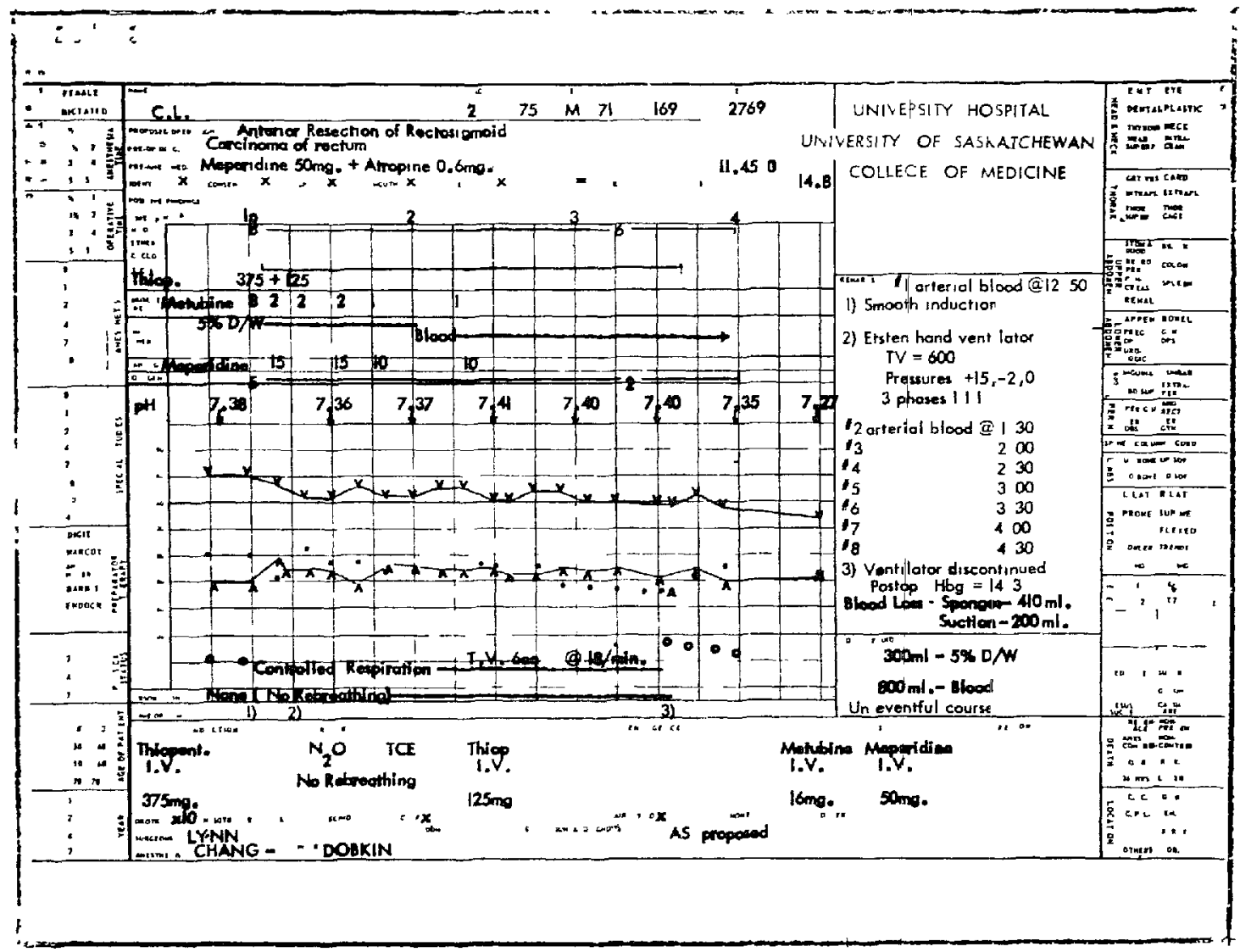

Figune 26. Anaesthetic chart. 
Discussion

\section{Effect of Anaesthetic Siystem}

Non-rebreathing system The small resistance, the negligible dead space and the elimination of rebreathing have the same advantages as have been described for children (39) The Fink type of non-rebreathing valve, in which the exhalation valve is occluded automatically during inhalation, makes the use of controlled respiration in the adult a much easier task (40). The operation of this valve is even more efficient when it is used with a positive and negative pressure ventilator Although the subatmospheric pressure is not transmitted to the patient's arrway, it serves to assure arrtight closure of the inhalation valve diaphragm during expıration and prevents stickıng of the inspuratory and expiratory valve diaphragms. One important point to remember about this valve, when used in conjunction with a fixed volume and pressure variable ventilator, is that the safety outlet must be closed tight to prevent escape of gas during inflation This outlet was left partially open inadvertently in a patient undergoing an extensive exploratory operation of the thoracic spine This leak in the system was sufficient to prevent adequate pulmonary ventilation Otherwise, pulmonary ventilation with this system was sufficient to mantain normal acid-base balance in every case.

The semi-closed system designed by Ivan Magill permits most of the gas expired by the patient to escape into the atmosphere through a pressure relief valve set close to the lips The remaning portion returns to mix with the oncoming flow of fresh gases The original purpose of this system was to conserve gas and to utilize the patient's exhaled carbon dioxide as a respiratory stımulant (1 e, increase the depth of breathing)

Several studies of this system during spontaneous respuration under anaesthesia have established that unless fresh gas in excess of the minute volume of respiration is delivered, progressive respiratory acidosis will develop (41-47). The average adult requires a flow of at least 8 litres When mechanical impedance affects the movement of the diaphragm or the intercostal muscles, even higher flows are insufficient.

When controlled respiration is employed with this system to overcome the mechanical impedance, it is difficult to know how much of the increased flow escapes through the pressure relief valve during inspiration The raised resistance to breathing would also tend to cause more gas to return to the rebreathing bag durng exprration. This system is therefore not as suitable for major or long procedures, in which respiration is controlled for the patient in an abnormal position because two undesirable defects are present-a large external dead space in the circuit and excessive resistance in the patient (48). Both of these enhance the tendency to respiratory acidosis. This explams why a mild respiratory acidosis developed in those patsents in the prone position, in spite of clinically satisfactory pulmonary ventilation

If it is necessary to conserve gas, the circle semi-closed system with carbon dioxide absorption should be employed. This means that trichlorethylene cannot be used, but this is not a great diawback as balanced anaesthesia may be carried on with intravenous analgesics (meperidine) or with Fluothane (0.3-05 per cent) together with nitrous, oxide-oxygen ( 21 ) 
The closed circle system came into widespread use with the introduction of cyclopropane $(49,50)$ This system was highly satisfactory, because it absorbed the expired carbon dioxide, conserved heat and water vapour, and permitted economy of anaesthetic gases (51)

Recent workers say that the canisters and the carbon dioxide absorbents generally used are inefficient (52-54) Several new styles of canısters and new brands of absorbents have been recommended These are sald to increase the efficiency of carbon dioxide absorbers in two ways by presenting a larger volume of absorbent to the expured gas, in order to reduce the rate of exposure, and permit a contunuous process of "regeneration", and by reducing channeling of the gases along the wall of the canister The latter is accomplished by coning the lower part of the canister or by inserting baffle plates

In this study, the older types of canister were used and were found to be efficient if fresh absorbent was properly packed in the canister, if the duration of use was limited ( 4 to 6 hours), and if rests (1\%-1 hour) were allowed during long anaesthetics A previous study of cyclopropane-oxygen in a closed curcle system with controlled respiration showed also that the $\mathrm{pCO}_{2}$ of patients in the supine position could usually be maintained at normal levels (55) There was no difficulty in maintainıng normal acid-base homeostasis in the closed system, even with the abnormal postures reported here

The conclusion to be drawn is no matter how efficient the absorption system, the $\mathrm{pH}$ will fall and the $\mathrm{pCO}_{2}$ will rise if ventlation is not adequate With adequate pulmonary ventilation, only poor quality absorbent, inefficient valves (high resistance) or leaks in the system will cause resprratory acidosis to develop

\section{Effect of Posture}

The effect on pulmonary ventilation of various postures employed for the convenience of the surgeon has been reviewed in previous publications $(16,56)$. The change from erect to the recumbent posture only slightly decreases total lung capacity, vital capacity and residual volume, but there is a large decrease in expiratory reserve volume with an inadequate compensatory increase in the inspiratory reserve volume Pulmonary mixing is also less adequate in the supine position In the lateral position, the lower lung has a decreased expiratory reserve volume When cardiopulmonary disease is present, there is a marked decrease in pulmonary compliance and increased mechanical resistance of the lungs. The resistance to air flow is considerably higher during expiration than during inspiration in all subjects (57-60)

These physical changes in the lungs are aggravated by anaesthesia, as there is even less change in volume with change in arrway pressure This effect is exaggerated if the patient is obese, or in an abnormal posture. In the lateral flexed position for thoracotomy the main impedance is on the lower lung which must perform the greater part of the ventilation since the lung on the side of operation is partially, or in some cases, entirely collapsed In the prone jackknife position, the difficulty is not only one of ventilation but also, unless the abdomen and chest are relatively free from pressure, there is marked increase in venous pressure which causes difficulty owing to persistent bleeding in the operative 
field (61) The head low-lithotomy position, as is required for combined abdominal perineal resection, appeared to have little or no effect, even in the presence of emphysema if the patient was not obese. Expiration was not hindered by abnormal postures as compression of the chest or abdomen assists this phase

\section{Effect of Airway Pressures}

In this study it was found that an inspiratory peak pressure of $15 \mathrm{~mm} \mathrm{Hg}$ was necessary in most of the patients to maintain adequate pulmonary ventilation with the rates and volumes selected This pressure had no deleterious effect on cardiovascular homeostasis, provided that the positive prassure phase (inspiration) did not last longer than one-third of the cycle, and the airway pressure dunng the expiratory phase remained at or below the ambient pressure in both the open chest and closed chest operations (15). The short period of subatmospheric pressure of low amplitude following inhalation appeared to support the blood pressure during the abdominal operations.

\section{Automatic Cycling of the Mechancal Ventilator}

When the anaesthetist is contronted by a patient who poses difficulties in ventilation owing to obesity, emphysema, or abnormal postures, an efficient automatic mechanical device removes a major bane In this situation the ventilator is as helpful to the patient and the anaesthetist as a self-retaining retractor is to the surgeon All other things being equal, any prolonged anaesthetic, particularly when the surgical requirements call for controlled respiration, is much better managed with an efficient automatic ventılator When applied to the non-rebreathing or closed circle system, after the volume and pressure in the anaesthetic circuit had been properly regulated, the Etsten ventilator provided adequate ventılation as efficiently when operated by the motor as by hand The only deficiency suspected, but not specifically observed with the pneumatic piston motor used in this study, was the need for a longer period for exhalation in the patients with severe emphysema.

\section{Clinical Signs and Mechanical Indicators}

Many warning signs precede the induction of anaesthesia, and place the anaesthetist duly on guard (62). It is unfortunate, however, that serious deficiencies in anaesthetic management produce few signals. The anaesthetist must therefore rely on prevention, rather than treatment. When signs (cold sweat, pallor, cyanosis, tachycardia, hypotension, tracheal tug, etc.) do appear, treatment is almost always instituted in the face of an impending catastrophe.

Adequate pulmonary ventilation is the most important requisite of a smooth anaesthetic for major operations. It is also the most difficult parameter to define in the operating room when the airway pressure and tidal volume are not monitored (63). Some physical methods for detecting acid base derangements due to inadequate pulmonary ventilation (respiratory acidosis) in its early stages have been described. There is a correlation between the level of the arterial blood $\mathrm{pCO}_{2}$ and the presence or absence of spontaneous respiratory efforts durng surgical pneumothorax When the tension of carbon dioxidé is elevated, 
spontaneous respiratory efforts are made whenever there is a pause in the controlled ventilation administered by the anaesthetıst, whereas no spontaneous respiratory efforts are made when the $\mathrm{pCO}_{2}$ is normal or low (64). This is a reliable sign, and is a guide to remember in all major operative procedures when controlled iespiration is employed The only time in the anaesthetic period when it cannot be relied upon is when anaesthesia is lightened as, for example, at the end of the operation. Then the patient must be allowed to "take over." The only course open to the anaesthetist at this time is to prevent hypoxia from occurring along with the tendency toward respiratory acidosis, as this combination is undoubtedly a primary cause of severe myocardial depression (65). In order to avoid this depression, it appears most efficacious to induce a mild respuratory alkalosis durng anaesthesia, and during the concluding minutes of the operation to allow the patient to breath spontaneously (even though inadequately at first) while oxygen only is administered. In this way, diffusion hypoxia is less likely to accompany the rise of $\mathrm{pCO}_{2}$ to normal or slightly elevated levels

The force of expiration is another important sign of pulmonary ventilation which has been mentioned This phase of the respiratory cycle should be carefully observed, for the development of "exprratory dominance" may be an important sign of hypoxia and hypercarbia (66), and is therefore an indication of inadequate pulmonary ventilation in the patient who is breathing spontaneously. Morton studied the force of expuration as a sign of anaesthesia, and found that when expiratory force decreased there was primarly evidence of severe depression of pulmonary ventilation by deep anaesthesia (67) During intrathoracic operations it was found also to be evidence of trapping of air in the lungs, even when automatic positive and negative pressure respiration is employed. Trapping leads rapidly to hypoxia, hypercarbia and impedes the work of the surgeon (16).

The resistance to air flow is an important consideration in setting the volume and pressure amplitude of a ventilator, especially in patients with emphysema, bronchitis and asthma. The resistance to arr flow during quiet inspuration is normally less than $2 \mathrm{~cm} . \mathrm{H}_{2} \mathrm{O} / \mathrm{L}$. $/ \mathrm{sec}$. This resistance is increased four times in the emphysematous patient and almost ten times in the asthmatic patient (6871 ). Since the resistance to arr flow is greater during expuration, it is understandable why a forced expiratory effort, or the applacation of excessive subatmospheric pressure to the airway durng anaesthesia, will produce, by the trapping phenomenon, a level of intrathoracic pressure which might plateau and even decrease the flow rate.

Light general anaesthesia with muscle relaxants for abdominal operations is sometimes inadequate, and is often accompanied by a variety of untoward effects, such as sudden hypotension, pallor and sweating, and postoperative mental exhaustion (72) With balanced light anaesthesia (hypnosis, analgesia and muscle relaxation) and concurrent replacement of fluid losses, judicious use of atropine and adequate pulmonary ventilation, these symptoms are not seen as commonly

From this study it is evident that the best guide to prevention of hypoxia and 
acıdosis is to provide a known tidal volume, rate of respiration, and airway pressure determmed accolding to the "normal" body weight and modified according to vanous impedances to ventlation (16,73-77).

\section{Summary and Conclusions}

Pulmonary ventilation was controlled mechanically and automatically in over 300 selected major operative procedures in order to decide upon the efficacy of regulatng such control by a predetermined volume and pressure amplitude. The volume selected was derived from Radford's nomogram, modified according to the "normal" weight for the patient as noted in a table of heights and weights related to the age group, and corrected for abnormal postures and the presence of pulmonary emphysema The pressure amplitude was mantaned as uniform as possible and was selected on the basis of previous experience with sumilar mechanical respirators A three-phase cycle, lasting about three seconds, consisting of an initial accelerated rise to peak inspiratory pressure lasting approximately one-third of the cycle, followed by a short period of subatmosphenc pressure and the remainder at atmospheric pressure was found to approach the ideal clinical condition most closely $(15,78,79)$.

These characternstics of the ventilation cycle were applued mainly to the three commonly employed anaesthetıc circuits the non-rebreathing system employing a senes of non-return vallves, including the Fink-type va ve, the Magll or Mapleson A type of semı closed system, and the closed curcle system. Nitrous oxıde was the primary anaesthetic mantenance agent, with supplements of trichlorethylene, mependine (intravenously) or occasionally a trace of ether, when the first two systems were used Cyclopropane was the primary maintenance agent, usually with a trace of ether, when the closed crrcle system was employed In every case, non-distensible tubing connected the anaesthetic machine to the patient and to the ventilator in order to ensure the delivery of the volume as set This volume was checked with a ventzlation meter, and was found to agree within the permissible volume error of the meter

The first criteron for deciding whether such control of pulmonary ventilation was satısfactory depended upon data derived. from arterial blood samples, drawn anaerobically from a representative number of patients after premedication, during the course of the anaesthetic and in the immediate postanaesthetic period. These were compared with data denved from several normal healthy adults, and from several patients whose respiration was controlled by manual compression of the rebreathing bag The blood data meassured directly included $\mathrm{pH}$, haematocrit, total carbon dioxide content, oxygen capacity, oxygen content and oxygen saturation. From these were derived the haemoglobm content, plasma $\mathrm{CO}_{2}$ content, plasma bicarbonate content, carbon dioxide tension and oxygen tension

The second criterion cepended upon the clinical observations during each individual anaesthetic as to the general condition of the patient durng and after the operation, and the facility of mantanning cardıovascular homeostasis. 
Whenever the non-rebreathing system was employed, anaesthesia was easy to maintain at levels of hypnosis, analgesia and muscle relaxation which were satisfactory to the surgeon and the anaesthetist. The general condition of the good risk patients was not affected adversely, when the patients were serıously ill on arrival for operation, their condition did not deteriorate and in most instances improved. Pulmonary ventilation, according to the blood data, was adequate in every case but one, in which an error in the mechanics of the non-rebreathing valve was overlooked Although a slight respiratory alkalosis developed in the majority of the patients managed with this system, there was no evidence of a compensatory metabolic alteration in the fixed acids, the respiratory alkalosis disappeared promptly, immediately after anaesthesia, and the return of spontaneous respiration was never delayed because of the method of ventilation These conditions prevailed in every type of operation that was studied with the non-rebreathing system

When the semi-closed system was employed, with nitrous oxide, meperidine and trichlorethylene, pulmonary ventilation was adequate in $t$ lose patients whose chest mobility was not impaired or hampered by abnorma postures. In the prone jackknife position and in the head low position, there wes a slight but definite trend toward respuratory acidosis which continued into tie postanaesthetic period This alteration was not accompanied by change in fixed acids Moderate but persistent hypotension and a small pulse pressure were usually seen with the prone jackknife position The hypotension extended to the postoperative period only when this system was used

The closed circle system employed ${ }^{n}$ with cyclopropane and a trace of ether, provided satisfactory operating conditions, and pulmonary ventılation in each case maintained acid base homeostasis Only with moderately deep ether anaesthesia could a mixed respiratory and metabolıc acidosis be overlooked in this system (80).

It was evident from these data that respiratory disturbances of acid base balance and disturbances of cardiovascular homeostasis need not occur during clinical anaesthesia, if adequate pulmonary ventilation is provided. It appears evident also that metabolic acidosis does not always accompany respiratorv acidosis, and does not appear when trace amounts of diethyl ether are used with nitrous oxide or cyclopropane With a non-rebreatlung system, all non-return valves and the expiratory valve must function perfectly; a semi-closed system is suitable only if high gas flows can be provided and abnormal postures are not required, and the closed circle system is highly efficient if the carbon dioxide absorbent is packed properly in the canister and the valves function with very low resistance The dictum of Leigh that "Careful observation both of the respiration and of the signs of its inefficiency is the most important function of the anaesthetist" (81) bears re-emphasis, but to it one must now add the statement: in major operative procedures, pulmonary ventilation should be controlled according to a predetermined volume and pressure amplitude according to the individual patient, to the anaesthetic crrcut employed, and to the posture adopted 


\section{ACKNOWLEDGMents}

I am indebted to Miss Bertha Cox, who carried out the analysis of the blood samples, to N. Shklov, Ph.D. for statistical analysis of the data; and to Dr. H. F. Poppelbaum of Strallsund, Germany, for permission to publish the diagram of his, ventilator

\section{RÉSUMÉ}

Sur au-délà de 300 cas, nous avons contrôlé, mécanıquement et automatıquement, la ventılation pulmonaure au cours d'opérations majeures afin d'apprécier l'efficacité d'un tel contróle dont le volume, la pressıon et l'amplitude étaient déerdés au préalable. On a cholsı le volume d'après le monogramme de Radford et calculé d'après le pords "normal" du malade tel que mentionné dans une table de tailles et poids selon l'âge, et corrıgé selon les positions anormales et la présence d'emphysème pulmonarre Nous avons conservé, autant que possible, un volume et une pression uniformes que nous avons déterminés d'après notre expérience acquise avec de semblables respirateurs mécanique Un cycle à trois phases, d'une durée approximative de trois secondes, consistant en une élévation rapıde initıale vers une pıession inspıratore maxımale d'une durée égale environ à un tiers du cycle, suivı d'une courte période de pressıon inféneure à la pressıon atmosphérique, et le reste du cycle à une pression égale à la pression atmosphérique nous a parụ réalıser à très peu près, la ventılatıon clinique idéale $(15,78,79)$

Nous avons étudié ces caractéristıques du cycle de ventılation en employant les trois différents circuits d'usage courant en anesthésıe en évitant la rémspiration au moyen d'une sérıe de valves, dont la valve du type de Fink, le système semı-fermé de Magill ou de Mapleson A et le système à crrcuit fermé. Nous avons employé comme agents avec les deux premiers systèmes de circuit le protoxyde d'azote comme agent principal, avec des traces de trichlorethylène, de la meperidine (1 v) et, à l'occasıon, des traces d'éther Lorsque nous avons employé le crrcuit fermé, nous avons utilisé le cyclopropane comme agent princrpal et, habituellement, un peu d'éther. Dans tous les cas, de façon à assurer que le volume déterminé était porté au malade, nous avons employé des tubes à paroıs non extensibles entre le malade, la machıne et le ventilateur. On à vérnfié ce volume par un ventılomètre et on n'a trouvé qu'une marge d'erreur admissible dans de pareilles curconstances.

Comme premier critère pour établir l'efficacité satısfausante de la ventılation pulmonaire contrôlée, nous avons comparé les résultats obtenus des analyses du sang artériel prélevé à l'abri de l'aur chez un bon nombre de malades, après la prémédicatıon, au cours de l'anesthésıe et ımmédiatement. après l'anesthésıe. Nous avons comparé ces résultats avec ceux obtenus chez des adultes en santé et chez plusieurs malades dont la respiration était contrôlée par compression manuelle sur le ballon. On a cherché dans le sang. le $\mathrm{pH}$, l'hématocrite, le contenu total de gaz carbonique, la capacité en oxygène, le contenu en oxygène et la saturation en oxygène. De ces données, on a trouvé. l'hémoglobine, le gaz 
carbonique plasmatıque, le bicarbonate plasmatıque, la tension de gaz carbonique et d'oxygène.

Comme deuxième critère, nous avons accepté les observations clnniques faites durant l'anesthésie de chacun des malades portant sur l'état général de chacun au cours de l'opération et après l'opération et sur la facilité de maintenrr l'équilıbre cardiovasculaire

A chaque fois que nous avons employé un système sans rémspiration, nous avonş réussı à maintenır facılement les nıveaux d'anesthésie désirés par le chırurgien et l'anesthésıe, soit pour le sommeıl, l’analgésie, ou le relâchement muscularre L'état général des malades en bonne santé n'a pas été touché outre mesure, l'état des malades séneusement touchés à l'arrivée à la salle d'opératıon ne s'est pas aggravé et, dans bien des cas, s'est amélioré D’après les résultats des analyses de sang, la ventilation pulmonaure est demeurée satisfaisante à l'exception d'un cas où ưne erreur s'est glıssée dans la mécanıque des valves En dépit du fait que nous' ayons observé une légère alcalose respiratorre chez la plupart des malądes trartés de cette façon, nous n'avons pas noté de réact ons métaboliques compensatrices dans les acides fixes, l'alcalose respiratoure est disparue rapidement, aussitôt après l'anesthésie, et le retour de la respiration spontanée n’a jamars été tardıf à cause de ce système de ventllation. Cet état de chose a été observé au cours de toutes les opératıons où le système de non-rémspiration a été étudié.

Quand nous avons employé un système semı-fermé, avec le protoxvde, la meperidine et le trichlorethylène, chez les malades où la ventılation pulmonaire n'était pas modifiée ou inhibée par les positions anormales qui agissent sur la mobilité thoracique, la ventilation pulmonaire est demeurée adéquate En position ventrale avec flexion et en position de Trendelenbourg, il est apparu une légère mass constante tendance à l'acıdose respiratoire qui a persisté au cours de la période post-anesthésıque Toutefołs, cela n'a pas modifié les acides fixes En position ventrale avec flexion, on a noté une hypotension légère mais persistante ainsi qu'une différentrelle pincée L'hypotension a persisté jusqu’à la période postopératorre seulement lorsque ce système a été employé

Avec l'emploi du crrcuit fermé, du cyclopropane et d'un peu d'éther, dans tous les cas, nous avons obtenu une anesthésie satısfaisante au cours des opérations et le mantien de l'équilbre acıde-base C'est seulement avec une anesthésie à l'éther plutôt profonde que nous avons constaté une acıdose respiratorre et métabolique avec ce système (80)

Il devient évident, d'après ces résultats, qu'on ne devrast pas observer, durant l'anesthésie clınıue, de modıficatıons de l'équilıbre acıde-base nı de modıficatrons de l'équilibre cardio-vasculaire, si l'on mantıent une ventilation pulmonarre adéquate Il appert également que l'acidose métabolıque n'accompagne pas toujours l'acidose respiratorre et qu'elle n'apparant pas lorsqu'on emploie des traces d'éther diéthylique avec le protoxyde ou le cyclopropane. Quand on emploie un système de non-rémspiration, toutes les valves empêchant le retour et la valve d'expiration doıvent fonctionner d'une façon parfaile Il convient d'employer le système semi-fermé seulement si l'on peut donner un grand débit 
de gaz et si la chirurgie n'exıge pas de position anormale Quant au circut fermé, ll est tout-à-fait efficace à condition que l'absorbant du gaz carbonıque soit bien tassé dans le contenant et que les valves n'ofirent qu'une résıstance minime Le dicton de Leigh. "une observation attentive de la respuration et des sıgnes de son efficacité demeure la fonction la plus importante de l'anesthésıste" (81) mérite qu'on insiste, mars, mantenant, il faut luı ajouter "au cours des opérations majeures, la veritılation pulmonaure devralt être contrôlée à un volume, une pression et une amplitude déterminés d'avance pour chacun des malades, selon la variété de circuit anesthésıque employé et la positıon exigée."

\section{REFERENCES}

I Stehle, R L, \& Bourne, W Mechanım of Acidosis in Anesthesia J Biol Chem 60 $17(1924)$

2 Waters, R M Carbon Dioxide Canad II A J 38240 (1938)

3 BeEchen, H K, \& MUrphy, A J Acidosis during Thoracic Surgery J Thoracic Surg. 1950 (1950)

4 Beecher, H. K, Francis, L, \& Anfinsen, C B Metabolic Effects of Anesthesia in Man I Acıd-Base Balance during Ether Anesthesia J Pharmacol \& Exper Therap. $9838(1950)$

5 Bunker, J P, Beecher, $\mathrm{H}$ K, Briggs, $\mathrm{B}$ D, Brewster, $W \mathrm{R}$, \& Barnes, B A Metabolic Effects of Anesthesia II A Comparison of Acid-Base Equlibrium in Man and in Dogs during Ether and during Cyclopropane Anesthessa J Pharmacol \& Exper Therap. $102 \cdot 62(1951)$.

6 Brewster, W R, JR, Buvker, J P, \& Beecher, $\mathrm{H} \mathrm{K}$ Metabolic Effects of Anesthesia VI Mechanım of Metabolic Acıdosis and Hyperglycemıa during Ether Anesthesia in the Dog Am J Physiol 17137 (1952)

7 Moore, F D, \& Ball, M R The Metabolic Response to Surgery Sprngfield, Ill C C Thomas (1952)

8 Moone, F D Bodily Changes in Surgical Convalescence I The Normal Sequence Observations and Interpretations Ann Surg 137289 (1953)

9 Moore, F D Hormones and Stress Endocrine Changes after Anesthesia, Surgery and Unanesthetzed Trauma in Man Recent Progress in Hormone Research 13511 (1957)

10 Holadax, D A, MA, D, \& Papper, E M The Immediate Effects of Respiratory Depression on Acid-Base Balance in Anesthetzzed Man J Cin. Invest 361121 (1957)

Il Cullen, S. C,; Comroe, J H, Jr, Brown, F B, Jr, Bezcher, H. K, \& Maloney, $\mathrm{J}$ V, Jr Problems in Ventlation Anesthesiology 15 416 ( 1954)

12 Mørach, E 'T , \& Benson, D. W. Automatic Artficial Respiration durng Anesthesia. Proc 3rd. Cong scand soc. Anaesth, p 30 (1954)

13 Alcibritten, F F, JR, Haupt, $G$ J, \& Aaradeo, J H The Change in Pulmonary Alveolar Ventilation Achieved by. Alding the Deflation Phase of Respiration durng Anesthesia for Surgical Operations Ann Surg 140569 (1954)

14. Nealon, T F, Jr, Hauft, G J , Price, J E, \& Grbbon, J H, Jr Pulmonary Ventilaton during Open Thos acotomy. Inflation and Deflation Ratios and Pressures $\mathrm{J}$ Thoracic Surg 30665 (1955)

15 Dobkin, A B , Hubay, C'A, Mendersogn, H J \& Hingson, R A Anaesthesia with Controlled Positive and Negative Pressure Respiration I A Clnical Evaluation Brit J Anaesth 28297 (1956)

16 Dobkin, A B \&.Wyant, G M Anaesthesia with Controlled Positive and Negative Pressure Respiration. II Review of Cardiorespiratory Function and Acid-Base Homeostasis Brit J Anaesth 28353 (1956) 
17. Nealon, T F, Jr, Haupt, G J , Chase, H F, Price, J E, \& Gibbon, J H Inefficient Carbon Dioxide Absorption requiring Increased Pulmonary Ventllation during Operations. J. Thoracic Surg 32464 (1956)

18 Matas, $R$ Ann Surg 29426 (1899) Quoted by Mushin, W W and Rendeil-Baker, L, in The Prnciples of Thoracic Anaesthesia Past and Present, p 44 Sprngfield, Ill C C Thomas (1953)

19. Poppel,baum, F Uber Wechseldruckbeatmung in der transpleuralen Lungenchirurgie Deutsche Ges Wesen. 26890 (1956)

20 Mackay, I M. A New Manual Respirator Canad Andesth Soc J 3113 (1956)

21 Bardees, A. A Special Pad for Patients in the Prone Position Anesthesiology 16465 (1955).

22 Singer, R B, \& Hastings, A B Impreved Chemical Method for Estimation of Disturbances of Acid-Base Balance in Human Blood Medıcine 27223 (1948).

23 Rosenthal, T B. The Effect of Temperature on the $\mathrm{pH}$ of Blood and Plasma in utro J. Biol Chem 17325 (1948)

24 Wuson, R H pH of Whole Arternal Blood. J Lab \& Clin Med 37129 (1951)

25 Roughton, F J W, Darimg, R C, and Root, W S Factors affectung the Determination of Oxygen Capacity, Content and Pressure m Human Arterial Blood Am J Physiol. 142708 (1944)

26 VAN Styke, D D, \& Neill, J. M. Determmation of Gases in Blood and Other Solutions by Vacuum Extraction and Manometric Measurement J Brol Chem 6 523 (1924)

27 Goldstemn, F, Gibbon, J H, Jr, Allbritten, F F, Jr, \& Stayman, J W, Jr The Combined Manometric Determination of Oxygen and Carbon Dioxide in Blood in the Presence of Low Concentrations of Ethyl Ether. J Biol Chem 182815 (1950)

28 Holaday, D A, \& Veroskr, M The Manometric Analysıs of Respuratory Gases in Blood containing Volatile Anesthetic Agents I Comparison of Macromethods J Lab \& Clin Med 45149 (1955)

29 Van Slyke, D D, \& Sendroy, J, Jn Line Charts for Graphic Calculations by HendersonHasselbalch Equation and for Calculating Plasma $\mathrm{CO}_{2}$ Content from Whole Blood Content (\#287). J Biol Chem. 79781 (1928).

30 Dill, D B, Eowards, H T, \& Consolazio, W V Blood as a Physicochemical System XI Man at Rest J Biol Chem $118^{\circ} 635$ (1937)

30a Dilx, D B Oxygen Dissociation Curves for Human Blood Handbook of Respiratory Data in Aviation. Wnght Field Aeromedical Unit, Washington, D C (1944)

31 Hickman, J B, \& Frayser, R Spectrophotometric Oxygen Determination on Whole Blood Samples Apparent Increase in Oxyhemoglobin under High Oxygen Tensions J Appl. Physiol 5 125 (1952-3)

32 Natas, G. G. Rapid Determination of Hemoglobin and Oxyhemoglobin in Whole Hemolyzed Blood, Using a Lucite Cuvette Adapted to the Beckman Spectrophotometer Amer J Physiol 163737 (1950)

33 Holling, H E, Macdonald, I, O'Halloran, J A, \& Venner, A Reliability of a Spectrophotometric Method of Estmating Blood Oxygen J Appl Physiol 8249 (1955).

34 Henderson, L J Blood A Study in General Physiology New Haven Yale Unıv Press (1928)

35. Davenport, H W. The A B C of Acid-Base Chemistry. Thurd edition Chicago Univer Chicago Press (19,50).

36. Mmch, $\mathrm{R}$ A, Bane, $\mathrm{H}$ N, \& Roberts, $\mathrm{K}$ E Conversion Factors for Serum Base-Bound Bicarbonate and Carbon Dioxide Tension J Appl Physiol. 10151 (1957)

37 Radford, E P, Jr., Ferris, B G, Jr, \& Khiete, B C. Cluncal Use of a Nomogram to Estımate Proper Ventilation during Artificial Respiratıon New England I Med 251. 877 (1954).

38. DoBkn, A. B. Chapter- "Pulmonary Ventulation and Its Control" in Modern Trends in Anaesthesia Edited by F. T. Evans \& T. C Gray. London Butterworth (in press) 
39 StepheN, C R, \& Sliter, H M A Non-Resistung, Non-Rebreathing Valve Anesthesiology 9550 (1948)

40 Fink, B R A Nonrebreathing Valve of New Design Anesthesiolozy 15 471 (1954)

41 Schwartz, C H, Alexander, M H, \& Adriani, J Semiclosec Inhalers Studies of Oxygen and Carbon. Dioxide Tensions during Various Conditions of Use Anesthesiology 14. 437 (1953).

42 Mapleson, W W The Elimination of Rebreathing in Various Sem1-Closed Anaesthetic Systems Bnt J Anaesth 26323 (1954)

43 Harrison, G G, \& Jones, C S Carbon Dioxide Concentration in the Boyle's Type Anaesthetic Machine Brit J Anaesth 27428 (1955)

44 Bracken, A, \& Sanderson, D M Carbon Dioxide Concentrations Found in Vanous Anaesthetic Circuits Brit T. Anaesth 27162 (1955)

45 Johnson, B D Carbon Dioxıde Concentration in Varıous Anaesthetıc Techniques Brit J Anaesth 2820 (1956).

46 Elan, J. O, \& Brown, E S Carbon Dioxide Homeostasis durng Anesthesia IV An Evaluation of the Partial Rebreathing System Anesthesiology 17128 (1956)

47 Bracken, A Discusşion on Carbon Dioxide Accumulation in Anaesthetic Circuits Proc Roy Soc Med 49215 (1956)

48 Clappison, G B, \& Hamilon, W K Respiratory Adjustments to Increases in External Dead Space Anesthesiology 17643 (1956)

49 Waters, $R$ M Adventages and Technque of Carbon Dioxide Filtration with Inhalation Anesthesia Anesth \& Analg 5 160 (1926)

50 Swond, B C The Closed Circle Method of Adminstration of Cias Anesthesia Anesth \& Analg 9198 (1930)

51 Adriani, J, \& Rovenstine, E A Experumental Studies on Carbon Dioxide Absorbers for Anesthesia Anesthesiology 2 1 (1941)

52 Mousel, L H, Weiss, W A, \& Gilliom, L A A Clincal Study of Carbon Dioude Absorption durung Anesthesia Anesthesiology 7375 (1946)

53 Prres, F K Chemical Control of Soda Lime Efficiency in Circle Filters Brit J Anaesth 2543 (1953)

54 Lund, I, Anderson, K L, \& Erikson, H Efficiency of Carbon Dioxide Absorption by Soda Lime in a Closed System Bnt T Anaesth 2813 (1956).

55 Buckley, J J, Van Bergen, F H, Dobkin, A. B, Brown, E B, Jr, Mrller, F A, \& VARCO, R L Postanesthetic Hypotension following Cyclopropane Its Relationship to Hypercapnia Anesthesiology 14226 (1953)

56. Henschel, A B, Wyant, G M, Dobkin, A B, \& Henschel, E O Posture as It Concerns the Anesthesiologist A Preliminary Study Anesth \& Analg 3669 (1957)

57 Attinger, E O, Monroe, $\mathrm{R}$ G, \& SEgAL, M S The Mechanics of Breathing in Different Body Positions I In Normal Subjects J Clin Investigation 35904 (1956)

58 Attinger, E O, Herschfus, J. A, \& Segal, M S The Mechanics of Breathing in Different Body Positions II In Cardiopulmonary Disease. J Chn Investigation 35 912 (1956)

59 Christie, C D, \& Bears, A J The Estimation of Nomal Vital Capacity with Especial Reference to the Effect of Posture Arch Int Med 3034 (1922)

60 Barach, A L., \& BECK, G J The Ventilatory Effects of the Head-down Position in Pulmonary Emphysema Am J Med 1655 (1954)

61 Pearce, D. J The Role of Posture in Lammectomy Proc Roy Soc Med 50109 (1957).

62 Eckenhofr, J E Some Preoperative Warmings of Potential Operatıng-Room Deaths New England J Med 2551075 (1956)

63 Scurr, C F Controlled Respiration Standardation of Ventulation Brit J Anaesth 28. $23(1956)$

64 Stead, W W, Martin, F E, \& Mrdplebrook, J Practucal Method for Detection of Early Respiratory Acidosıs during Thoracic Surgery. J Thoracic Surg 27306 (1954). 
65 FINK, B. R. Diffusion Anoxıd Anesthesiology 16511 (1955)

66. Moxer, C A, \& McKetrrick, J. B Practical Consideration of Open Pneumothorax under Sodium Evipal, Sodium Pentothal and Ether Anesthesia Ann Surg 116553 (1942)

67 Monton, H J. V. The Force of Expiration as a Sign of Anaesthesia Proc Roy Soc Med 38441 (1945)

68 Campbell, E J M, Mahtin, H B, \& Riley, R L M 6 chanisms of Airway Obstruction Bull Johns Hopkins Hosp 101329 ( 1957)

69 Dayman, $H$ Mechanics of Airflow in Health and in Emplaysema $J$ Clin Investigation $30 \quad 1175$ (1951)

70 Fry, D L, Ebert, R V, Stead, IV W, \& Brown, C C The Mechanics of Pulmonary Ventilation in Normal Subjects and, Patients with Emphysema Am J Med $16 \quad 80$ (1954)

71 Mead, J, Lindgren, I, \& Gaensler, E A The Mechanical Properties of the Lungs in Emphysema J Clin Investigation 341005 (1955)

72 Loder, $\mathrm{R}$ E Inadequacy of General Anaesthesia for Abdominal Operations A Possible Solution Lancet 2468 (1957)

73 Leigir, M D Acidosis during Clinical Anesthesia Anesthesiology 3429 (1942)

74 Hunt, K H Resistance in Respiratory Valves and C misters Anesthesiology 16190 (1955)

75 Lucas, B G B, \& Milne, E H Acid Base Balance and Anaesthesld. Thorax 10354 (1955)

76 SAmson, H H Hypoventilation Its Dangers in General Anesthesia South African M J $30 \quad 470(1956)$

77 Sieker, H O, \& Hickan, J B Carbon Dioxide Intoxication The Clinical Syndrome, Its Etiology and Managenent with Particular Reference to the Use, of Mechanical Respirators Medicine 35389 (1956)

78 Cournand, A, Motley, H L, Werko, L, \& Richards, D W, Jr Physiological Studies of the Effects of Intermittent Positive Pressure Breatlung on Cardiac Output in Man Am J Physiol 152162 (1948)

79 Gordon, A S, Frye, C W, \& Langston, H T The Cardiorespiratory Dynamics of Controlled Respiration in the Open and Closed Chest J Thoracic Surg 32431 (1956)

80 Fordhair, C C, \& Reilatan, A S Mined Respiratory and Metabolic Acidosis New England J Med 256698 (1957)

81 Leigh, M D Respiration during Anaesthesid Canad M A J 43 340 (1940) 